\title{
More on superstring chiral measures
}

\author{
Francesco Dalla Piazza ${ }^{a}$ \\ ${ }^{a}$ Dipartimento di Scienze Fisiche e Matematiche, Università dell'Insubria, Via \\ Valleggio 11, I-22100 Como, Italia
}

\begin{abstract}
In this paper we study the expressions of the superstring chiral measures for $g \leq 5$. We obtain certain new expressions which are functions of higher powers of theta constants. For $g=3$ we show that the measures can be written in terms of fourth power of theta constants and for $g=4$ in terms of squares of theta constants. In both cases the forms $\Xi_{8}^{(g)}\left[0^{(g)}\right]$ appearing in the expression of the measures are defined on the whole Siegel upper half space. Instead, for $g=5$ we find a form $\Xi_{8}^{(5)}\left[0^{(5)}\right]$ which is a polynomial in the classical theta constants, well defined on the Siegel upper half space and satisfying some suitable constraints on the moduli space of curves (and not on the whole Siegel upper half space) that could be a candidate for the genus five superstring measure. Moreover, we discuss the problem of the uniqueness of this form in genus five. We also determine the dimension of certain spaces of modular forms and reinterpret the vanishing of the cosmological constant in terms of group representations.
\end{abstract}

Key words: superstrings, amplitudes, modular forms, finite geometry, group representations, theta constants 1991 MSC: 83E30, 46T12, 14K25, 05B25, 20C15

\section{Introduction}

String theory in the perturbative approach can be formulated using the path integral formalism outlined by Polyakov. The starting point is the functional integral over all the fields in the theory, the embeddings and the metrics. Employing the symmetries

Email address: f.dallapiazza@uninsubria.it (Francesco Dalla Piazza). 
of the theory one reduces this infinite dimensional integral to a finite dimensional one over the moduli space of suitable Riemann surfaces. A Riemann surface can be recovered from its period matrix (see Appendix A). Expanding such an integral in series over the genus of the Riemann surfaces one can compute perturbatively the scattering amplitudes. This was emphasised in part by Belavin and Knizhnik [2] who conjectured that "any multiloop amplitude in any conformal invariant string theory may be deduced from purely algebraic objects on moduli spaces $M_{p}$ of Riemann surfaces". These amplitudes were computed in the 80's up to the four loop order for the bosonic string, and at zero and one loop for the supersymmetric case. In a series of papers D'Hoker and Phong determined an expression for the two loop superstring measure. Recently, in [7], a candidate was proposed for the three loop amplitude. In all these cases the measure is expressed in terms of suitable polynomials in the theta constants. The latter are defined on the Siegel upper half space $\mathbf{H}_{g}$ and not just on the subvariety $J_{g} \subseteq \mathbf{H}_{g}$ of period matrices of genus $g$ Riemann surfaces. This makes the superstring measure, for $g \leq 3$, a function over the whole $\mathbf{H}_{g}$. This fact is not completely surprising because, for $g \leq 3, \mathbf{H}_{g}$ and $J_{g}$ have the same dimension and $J_{g}$ is an open set of $\mathbf{H}_{g}$. In [8] we proposed an expression for the four loop superstring measure which again turned out to be defined over the whole $\mathbf{H}_{4}$; this is a remarkable fact, the dimension of the two varieties being no longer the same, see Table 1. In [17] a candidate was proposed for the superstring measure for any

\begin{tabular}{cccc}
\hline$g$ & $\operatorname{dim} \mathbf{H}_{g}$ & $\operatorname{dim} J_{g}$ & $\operatorname{codim}_{\mathbf{H}_{g}} J_{g}$ \\
\hline 2 & 3 & 3 & 0 \\
3 & 6 & 6 & 0 \\
4 & 10 & 9 & 1 \\
5 & 15 & 12 & 3 \\
$g$ & $\frac{1}{2} g(g+1)$ & $3 g-3$ & $\frac{1}{2}(g-2)(g-3)$ \\
\hline
\end{tabular}

Table 1

Dimensions of the varieties $\mathbf{H}_{g}$ and $J_{g}$.

genus $g$. However, these are not a priori well defined for $g \geq 5$ due to the presence of roots. In [33], it was proved that for $g=5$ this measure is well defined, at least on $J_{g}$. From these facts it is quite natural to investigate if the measure for $g=5$ could be extended, using the classical theta constants, over all $\mathbf{H}_{5}$ and what happens for $g>5$. Recently in [27] a candidate for the superstring measure for $g=5$ was proposed employing the notion of the lattice theta series. This formalism is almost equivalent to the one of the classical theta constants. Actually, the spaces spanned 
by theta series and the ones generated by the bases for the $\mathrm{O}^{+}$-invariants defined in Sections 3.1, 3.2 and 3.3 are the same. In genus five both formalisms lead to the same solutions, providing we add to the constraints also the request of the vanishing of the cosmological constant, as we have shown in [9].

The proposal for the $g$-loop superstring measure rests on the ansatz (not yet proved) of D'Hoker and Phong [12] that the genus $g$ vacuum to vacuum amplitude takes the form of an integral over the moduli space of genus $g$ Riemann surfaces of a suitable differential form that splits into a holomorphic and anti-holomorphic part. Moreover, the measure $d \mu\left[\Delta^{(g)}\right]$ should satisfy certain reasonable constraints. This characterises it uniquely for $g \leq 4$ (see below). In this paper we prove that, assuming these features for the amplitude, the superstring measure can be defined on the whole $\mathbf{H}_{g}$ for $g \leq 5$, but for $g=5$ the correct restriction (see point 3 of theorem 10) holds true just on $J_{4}$. This result is stated by the following:

Theorem 1 If the genus $g$ vacuum to vacuum amplitude takes the general form:

$$
\mathcal{A}=\int_{\mathcal{M}_{g}}(\operatorname{det} \operatorname{Im} \tau)^{-5} \sum_{\Delta, \bar{\Delta}} c_{\Delta, \bar{\Delta}} d \mu\left[\Delta^{(g)}\right](\tau) \wedge \overline{d \mu\left[\Delta^{(g)}\right](\tau)},
$$

where the form $d \mu\left[\Delta^{(g)}\right]$ can be written as:

$$
d \mu\left[\Delta^{(g)}\right]=c_{g} \Xi_{8}^{(g)}\left[\Delta^{(g)}\right]\left(\tau^{(g)}\right) d \mu_{B}^{(g)}
$$

and the functions $\Xi_{8}^{(g)}\left[\Delta^{(g)}\right]$ satisfy the following ansätze:

(1) they are holomorphic functions on $J_{g}$;

(2) under the action of $\Gamma_{g}=\operatorname{Sp}(2 g, \mathbf{Z})$ they should transform as $\Xi_{8}^{(g)}\left[M \cdot \Delta^{(g)}\right](M \cdot \tau)=\operatorname{det}(C \tau+D)^{8} \Xi_{8}^{(g)}\left[\Delta^{(g)}\right](\tau)$, for all $M \in \operatorname{Sp}(2 g, \mathbf{Z}) ;$

(3) the restriction of these functions to 'reducible' period matrices is a product of the corresponding functions in lower genus;

then $\Xi_{8}^{(g)}\left[\Delta^{(g)}\right]$, and so $d \mu\left[\Delta^{(g)}\right]$, are defined everywhere on $\mathbf{H}_{g}$, they can be expressed in terms of polynomials in square of theta constants and they are unique if $g \leq 4$. For $g=5$, for every even characteristic $\Delta^{(5)}$, at least one (actually many) forms $\Xi_{8}^{(5)}\left[\Delta^{(5)}\right]$ exist, it is defined on $\mathbf{H}_{5}$, it can be written as a polynomial in the theta constants and the restriction requested is satisfied just on $J_{4}$. The three constraints do not characterize it uniquely (at least on $\mathbf{H}_{5}$ ).

In the theorem the uniqueness for $g=4$ must be understood as uniqueness up to a multiple of $J^{(4)}$, that vanishes on the Jacobi locu $11 J_{4}$ (see below). In genus five,

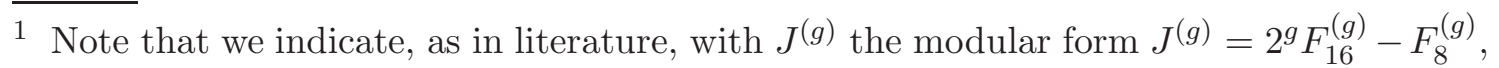


instead, the uniqueness is completely lost. One can find many forms $\Xi_{8}^{(5)}\left[0^{(5)}\right]$ that differ not just for something vanishing on the Jacobi locus. Actually, starting from a form $\Xi_{8}^{(5)}\left[0^{(5)}\right]$ satisfying the three constraints and adding a multiple of $J^{(5)}$, one obtains again a form satisfying the constraints. In genus five $J^{(5)}$ does not vanish on the Jacobi locus, see [18. It is still an open problem whether, adding to the constraints the request of the vanishing of the cosmological constant, the uniqueness of the measure is guaranteed. However, the vanishing of the cosmological constant should be automatic for a supersymmetric theory and not imposed by hand. At the moment it is not known if could exist some function satisfying the three constraints and differing from a $\Xi_{8}^{(5)}$ not just for a multiple of $J^{(5)}$. Moreover, nothing we can say if we consider also the non normal part of the ring of genus five modular forms.

Here $\Delta^{(g)}$ and $\overline{\Delta^{(g)}}$ denote two even genus $g$ theta characteristics, $c_{\Delta, \bar{\Delta}}$ are suitable constant phases depending on the details of the string model, $d \mu\left[\Delta^{(g)}\right](\tau)$ $\left(\overline{d \mu\left[\Delta^{(g)}\right](\tau)}\right)$ is a holomorphic (anti-holomorphic) form and $d \mu_{B}^{(g)}$ is the well defined genus $g$ bosonic measure. However, there is not an explicit form for $d \mu_{B}^{(g)}$ in higher genus. We observe that the transformation request for the form $\Xi_{8}^{(g)}\left[\Delta^{(g)}\right]$ is automatic for the integral to make sense, but, as usual, we prefer to emphasize this property for its crucial role in what follows.

For the genus two and three cases the uniqueness of the forms $\Xi_{8}^{(g)}\left[\Delta^{(g)}\right]$ is shown in [10]. In the same paper, assuming that the measure is a polynomial in the theta constants, the uniqueness (up to a term proportional to $J^{(4)}$ ) is shown for the genus four case and in [27] the general case is considered (see Section 6.1.1 below). In genus five the uniqueness can not be longer assured. Actually, in [27] a candidate for the genus five superstring measure is proposed. The authors make use of the notion of the lattice theta series. An analysis of the different expressions for the chiral superstring measure can be found in [14,21]. In a forthcoming paper [9] we will prove that the form $\Xi_{8}^{(5)}\left[0^{(5)}\right]$ defined there and the one defined in Section 7 are different on $\mathbf{H}_{5}$ and also on $J_{5}$. Actually, the difference is proportional to $J^{(5)}$. The supplementary request of the vanishing of the cosmological constant makes equivalent the two forms.

From the result of Salvati Manni [33, we know that the square root appearing in Grushevsky expression of the five loop measure (in the function $G_{5}^{(5)}\left[0^{(5)}\right]$ ) is well defined on the moduli space of curves $J_{5}$. Further investigations are needed to understand if, at least, on the locus of curves, it is polynomial in the classical theta constants.

see Section [3.4, and with $J_{g}$ the Jacobi locus. 
An indication that the three constraints cannot define the forms $\Xi_{8}^{(g)}\left[\Delta^{(g)}\right]$ defined over the whole $\mathbf{H}_{g}$ and are sufficient to assure their uniqueness comes from the increasing difference between the dimensions of $\mathbf{H}_{g}$ and $J_{g}$. The dimension of $\mathbf{H}_{g}$ is quadratic in $g$, instead the dimension of $J_{g}$ has a linear growth in $g$ and their difference is quadratic in $g$, see Table 1. Thus, it is not surprising that the constraints for the $\Xi_{8}^{(g)}\left[\Delta^{(g)}\right]$ are not strong enough to characterize it uniquely. Further investigations on this point will be carried on in [9].

\section{The strategy}

In [7], inspired by the factorisation of the superstring chiral measure at lower genus, a modification of the ans̈atze of D'Hoker and Phong was proposed for the superstring measure. Accordingly, the measure should be written as:

$$
d \mu\left[\Delta^{(g)}\right]=c_{g} \Xi_{8}^{(g)}\left[\Delta^{(g)}\right]\left(\tau^{(g)}\right) d \mu_{B}^{(g)}
$$

where $d \mu_{B}^{(g)}$ is the bosonic measure at genus $g$ and $\Xi_{8}^{(g)}\left[\Delta^{(g)}\right]$ are suitable functions, see below, $g$ is the genus of the Riemann surfaces considered and $\Delta^{(g)}$ is an even characteristic at genus $g$. The functions $\Xi_{8}^{(g)}\left[\Delta^{(g)}\right]$ are required to satisfy suitable transformation and factorisation constraints, see [7] Section 2.2, briefly summarised here:

(1) they must be holomorphic functions on $J_{g}$;

(2) under the action of $\Gamma_{g}=\operatorname{Sp}(2 g, \mathbf{Z})$ they should transform as $\Xi_{8}^{(g)}\left[M \cdot \Delta^{(g)}\right](M$. $\tau)=\operatorname{det}(C \tau+D)^{8} \Xi_{8}^{(g)}\left[\Delta^{(g)}\right](\tau)$, for all $M \in \operatorname{Sp}(2 g, \mathbf{Z})$;

(3) the restriction of these functions to 'reducible' period matrices is the product of the corresponding functions in lower genus.

Here we emphasise that the first constraint requires that the forms $\Xi_{8}^{(g)}\left[\Delta^{(g)}\right]$ are defined on the subvariety $J_{g} \subset \mathbf{H}_{g}$ of period matrices of Riemann surfaces of genus $g$ and not on the whole Siegel upper half space. In fact, $\operatorname{dim} \mathbf{H}_{g}=g(g+1) / 2$ and $\operatorname{dim} J_{g}=3 g-3$ so these two spaces are the same just for $g \leq 3$. Since we are interested in arbitrary genus, we write $J_{g}$ instead of $\mathbf{H}_{g}$. Actually, for $g \leq 4$ the superstring measure can be extended to the Siegel upper half space, instead for $g=5$ the forms constructed using the classical theta constants, although well defined over the whole $\mathbf{H}_{5}$, have the correct factorization just on the Jacobi locus $J_{4}$, see Section 7.2 . 
In [7] it was pointed out that the $\Xi_{8}^{(g)}\left[\Delta^{(g)}\right]$ are modular forms with respect to the normal subgroup $\Gamma_{g}(2)$ of $\operatorname{Sp}(2 g, \mathbf{Z})$ defined by:

$$
\begin{aligned}
\Gamma_{g}(2): & =\operatorname{ker}\left(S p(2 g, \mathbf{Z}) \longrightarrow S p\left(2 g, \mathbf{F}_{2}\right)\right) \\
& =\{M \in S p(2 g, \mathbf{Z}): A \equiv D \equiv I, B \equiv C \equiv 0 \bmod 2\}
\end{aligned}
$$

where $\mathbf{F}_{2}:=\mathbf{Z} / 2 \mathbf{Z}$ is the field of two elements. Furthermore we can restrict our attention on a single function (see, [7] Section 2.7) say $\Xi_{8}^{(g)}\left[0^{(g)}\right]$, where $\left[0^{(g)}\right]:=\left[\begin{array}{l}0 \cdots 0 \\ 0 \cdots 0\end{array}\right]$, which has to be a modular form of weight 8 on $\Gamma_{g}(1,2)$, where $\Gamma_{g}(1,2)$ is the subgroup of $\operatorname{Sp}(2 g, \mathbf{Z})$ which fixes the characteristic $\left[0^{(g)}\right]$. In general it is useful to define:

$$
\begin{aligned}
\Gamma_{g}(n, 2 n): & =\left\{M \in \Gamma_{g}(n): M \cdot\left[\begin{array}{l}
0 \\
0
\end{array}\right] \equiv\left[\begin{array}{l}
0 \\
0
\end{array}\right] \bmod 2 n\right\} \\
& =\left\{M \in \Gamma_{g}: \operatorname{diag} A^{t} B \equiv \operatorname{diag} C^{t} D \equiv 0 \bmod 2 n\right\} .
\end{aligned}
$$

Thus, the $\Xi_{8}^{(g)}\left[\Delta^{(g)}\right]$ are obtained by employing the transitive action of $\operatorname{Sp}(2 g, \mathbf{Z})$ on the even characteristics. The action of $M \in \operatorname{Sp}(2 g):=\operatorname{Sp}\left(2 g, \mathbf{F}_{2}\right) \cong \Gamma_{g} / \Gamma_{g}(2)$ on a characteristic $\Delta^{(g)}$ is given by:

$$
\left(\begin{array}{ll}
A & B \\
C & D
\end{array}\right) \cdot\left[\begin{array}{l}
a \\
b
\end{array}\right]:=\left[\begin{array}{l}
c \\
d
\end{array}\right], \quad\left(\begin{array}{c}
{ }^{t} c \\
{ }^{t} d
\end{array}\right)=\left(\begin{array}{cc}
D & -C \\
-B & A
\end{array}\right)\left(\begin{array}{c}
{ }^{t} a \\
{ }^{t} b
\end{array}\right)+\left(\begin{array}{c}
{ }^{t}\left(C^{t} D\right)_{0} \\
{ }^{t}\left(A^{t} B\right)_{0}
\end{array}\right) \quad \bmod 2,
$$

where $a, b, c$ and $d$ are the rows of $\Delta^{(g)}$ and $N_{0}=\left(N_{11}, \ldots, N_{g g}\right)$ is the row vector of diagonal entries of the matrix $N$.

As explained in [10], to which we refer for definitions and notations, the group $\Gamma_{g}$ acts on the $2^{2 g}$ points of $\mathbf{F}_{2}^{2 g}$ and on the characteristics through its quotient $\operatorname{Sp}(2 g) \cong$ $\Gamma_{g} / \Gamma_{g}(2)$. We defined the subgroup $\Gamma_{g}(1,2)$ of $\Gamma_{g} \equiv \Gamma_{g}(1)$ as the stabiliser of $\left[0^{(g)}\right]$ and the image of this subgroup in $\operatorname{Sp}(2 g)$ is called $O^{+}(2 g):=\Gamma_{g}(1,2) / \Gamma_{g}(2) \subset \operatorname{Sp}(2 g)$. The three requests, which the function $\Xi_{8}^{(g)}\left[0^{(g)}\right]$ should satisfy, imply that it must belong to the subspace of $\mathrm{O}^{+}$-invariants of weight 8:

$$
M_{8}\left(\Gamma_{g}(2)\right)^{O^{+}}:=\left\{f \in M_{8}\left(\Gamma_{g}(2)\right): \rho(h) f=f \quad \forall h \in O^{+}(2 g)\right\} .
$$

Here $M_{k}\left(\Gamma_{g}(2)\right)$ is the finite dimensional complex vector space of the Siegel modular forms of genus $g$, weight $k$ and level 2 and $\rho$ is the representation of the finite group $\operatorname{Sp}(2 g)$ on this space defined by:

$$
\left(\rho\left(h^{-1}\right) f\right)(\tau):=\operatorname{det}(C \tau+D)^{-k} f(M \cdot \tau)
$$


where $M \in \Gamma_{g}$ is a representative of $h \in \operatorname{Sp}(2 g)$ and $f \in M_{k}\left(\Gamma_{g}(2)\right)$. The action of $\Gamma_{g}$ on $\tau \in \mathbf{H}_{g}$, the Siegel upper half space, is given by:

$$
M \cdot \tau:=(A \tau+B)(C \tau+D)^{-1}, \quad M:=\left(\begin{array}{cc}
A & B \\
C & D
\end{array}\right) \in S p(2 g, \mathbf{Z}), \quad \tau \in \mathbf{H}_{g}
$$

Among the functions in $M_{8}\left(\Gamma_{g}(2)\right)$, we will search for the ones satisfying the three constraints. This is a general procedure, but for $g>4$ there are some subtleties due to the loss of the uniqueness of the form $\Xi_{8}^{(g)}\left[0^{(g)}\right]$. Let us briefly summarise these points, referring to [10] for more details. To build the $O^{+}$-invariants we use the $2^{g}$ second order theta constants, see [6] for details, defined by:

$$
\Theta[\sigma](\tau):=\theta\left[\begin{array}{l}
\sigma \\
0
\end{array}\right](2 \tau, 0), \quad[\sigma]=\left[\begin{array}{llll}
\sigma_{1} & \sigma_{2} & \ldots & \sigma_{g}
\end{array}\right], \sigma_{i} \in\{0,1\}, \tau \in \mathbf{H}_{g},
$$

where $\theta\left[\begin{array}{c}a \\ b\end{array}\right](\tau, z)$ are the usual Riemann theta functions. These theta constants are "modular forms of weight $1 / 2$ " on $\Gamma_{g}(2,4)$ and the invariants of degree $4 k$ of the quotients group $\Gamma_{g}(2) / \Gamma_{g}(2,4) \cong \mathbf{F}_{2}^{2 g}$ in the ring of polynomials in the $\Theta[\sigma]$ 's are modular forms of weight $2 k$ on $\Gamma_{g}(2)$. Indeed, due to the half integer weight, the group acting on the theta constants is the Heisenberg group, the central extension of the group $\Gamma_{g}(2) / \Gamma_{g}(2,4)$. Let us denote the space of Heisenberg invariants as $M_{2 k}^{\theta}\left(\Gamma_{g}(2)\right) \subset M_{2 k}\left(\Gamma_{g}(2)\right)$ and $M_{2 k}^{\theta}\left(\Gamma_{g}(2)\right):=\mathbf{C}[\ldots, \Theta[\sigma], \ldots]_{4 k}^{H_{g}}$, where $\mathbf{C}[\ldots, \Theta[\sigma], \ldots]_{4 k}$ is the subspace of homogeneous polynomials of degree $4 k$ in the $\Theta[\sigma]$ 's. It can be shown (cf. [34] Thm 2, [30], 31]) that any modular form of weight $2 k$ can be written as a homogeneous polynomial of degree $4 k$ in the $\Theta[\sigma]$ 's if $g \leq 3$, thus

$$
M_{2 k}^{\theta}\left(\Gamma_{g}(2)\right)=M_{2 k}\left(\Gamma_{g}(2)\right) \quad \text { for } \quad g=1,2,3 .
$$

Such a polynomial is unique for $g<3$, whereas for $g=3$ it is unique for degree at most than 15 otherwise it is determined up to the addition of $J^{(3)} G_{4 k-16}$ where $G_{4 k-16}$ is any homogeneous polynomial of degree $4 k-16$ in the $\Theta[\sigma]$ 's and $J^{(3)}$ is a polynomial of degree 16 vanishing identically on the $\Theta[\sigma]$ 's, see [36]. In all other genera there could exist some modular forms which cannot be written as polynomials in the $\Theta[\sigma]^{\prime} 2$.

The dimension of the space of $\mathrm{O}^{+}$-invariants can be determined from the decomposition of the $\operatorname{Sp}(2 g)$-representation into irreducible representations and using the Frobenius reciprocity. Thus, the dimension is given by the multiplicity of the trivial representation 1 of $O^{+}$in the $O^{+}$-representation $\operatorname{Res}_{O^{+}}^{\operatorname{Sp}(2 g)}(V)$ :

$$
\operatorname{dim} V^{O^{+}}=\left\langle\operatorname{Res}_{O^{+}}^{S p}(V), \mathbf{1}\right\rangle_{O^{+}}=\left\langle V, \operatorname{Ind}_{O^{+}}^{S p}(\mathbf{1})\right\rangle_{S p}
$$

$\overline{2}$ In general the graded ring of modular forms of even weight on $\Gamma_{g}(2)$ is the normalization of the ring of the $\Theta[\sigma]$ 's: $\oplus_{k=0}^{\infty} M_{2 k}\left(\Gamma_{g}(2)\right)=\left(\mathbf{C}[\ldots, \Theta[\sigma], \ldots]^{H_{g}}\right)^{N o r}$. 
Here $\operatorname{Res}_{O^{+}}^{S p}(V)$ is the restriction of the representation from $S p(2 g)$ to $O^{+}(2 g)$, $\operatorname{Ind}_{O^{+}}^{S p}(\mathbf{1})$ is the induced representation of the representation $\mathbf{1}$ of $\mathrm{O}^{+}(2 g)$ to the whole $S p(2 g)$ and the second identity is the Frobenius identity, see [10,6,32. Frame [16] showed that $\operatorname{Ind}_{O^{+}}^{S p}(\mathbf{1})=\mathbf{1}+\sigma_{\theta}$, where $\mathbf{1}$ is the trivial representation and $\sigma_{\theta}$ is an irreducible representation of dimension $2^{g-1}\left(2^{g}+1\right)-1$, so that if the multiplicities of 1 and $\sigma_{\theta}$ in $V$ are $n_{1}$ and $n_{\sigma_{\theta}}$ respectively, the dimension of the space of $O^{+}$-invariants is $\operatorname{dim} V^{O^{+}}=n_{1}+n_{\sigma_{\theta}}$.

\begin{tabular}{ccc}
\hline$g$ & $\sigma_{\theta}$ & $\operatorname{dim}\left(\sigma_{\theta}\right)$ \\
\hline 1 & $\rho_{[21]}$ & 2 \\
2 & $\rho_{[42]}$ & 9 \\
3 & $\mathbf{3 5}_{b}$ & 35 \\
4 & $\mathbf{1 3 5}$ & 135 \\
5 & $\mathbf{5 2 7}$ & 527 \\
\hline
\end{tabular}

Table 2

The $\sigma_{\theta}$ representations for the low genus cases and their dimensions.

In what follows we will label the irreducible representations of $\operatorname{Sp}(2 g)$ with the partitions of 3 and 6 for genus one and two respectively (recall that $\operatorname{Sp}(2) \cong S_{3}$ and $\left.\operatorname{Sp}(4) \cong S_{6}\right)$, as in [5]; we will follow Frame's notation [15] for genus three and indicate them just with their dimension 3 for $g \geq 4$. In Table 2 are reported the $\sigma_{\theta}$ representations for the lower genus cases. In case $g=1, \rho[21]$ is the unique two dimensional representation of $S_{3} \cong \operatorname{Sp}(2)$ and [21] is the partition of 3 labelling it. For $g=2, \rho[42]$, or $n_{9}$ in the notations of [5], is the nine dimensional representation of $S_{6} \cong \operatorname{Sp}(4)$ for which the character of $\mathbf{1}+\sigma_{\theta}$ is positive ([42] is the partition of six labelling this irreducible representation; see [10] Section 4.2 and [6] Section 5.2.1 for the explanation of why the character of the representation must be positive). For $g=3$, the $3 \mathbf{5}_{b}$ is the unique 35 dimensional representation of $\operatorname{Sp}(6)$, as reported in [15] or as can be computed using, for example, the software Magma. For $g=4$, 135 is the unique 135 dimensional irreducible representation of $\operatorname{Sp}(8)$ and for $g=5$, 527 is the unique 527 dimensional irreducible representation of $\mathrm{Sp}(10)$, as can be computed using Magma.

3 If they are not unique at the given size, we will indicate also the character of the second conjugacy class, the one of the non zero transvections (which has 255 and 1023 elements for genus four and five respectively). Transvections are analogous to reflections in orthogonal groups (cf. [20], § 6.9 or [10]). 
In [10, the representations of $\operatorname{Sp}(2 g)$ on $M_{k}\left(\Gamma_{g}(2)\right)$, the vector space of Siegel modular forms of given weight for the principal congruence subgroup of level two, were studied for small genus $g$, and decomposed into irreducible representations. For the applications in string theory, we are interested in the representations of $\operatorname{Sp}(2 g)$ on the space $M_{8}\left(\Gamma_{g}(2)\right)$, the modular forms of weight eight with respect to the group $\Gamma_{g}(2)$. Let us report here the decomposition of these representations for $g \leq 3$ :

$$
\begin{aligned}
M_{8}\left(\Gamma_{1}(2)\right) \cong \operatorname{Sym}^{4}\left(\rho_{[21]}\right)=\mathbf{1}+2 \rho_{[21]}, \\
M_{8}\left(\Gamma_{2}(2)\right) \cong \operatorname{Sym}^{4}\left(\rho_{\left[2^{3}\right]}\right)-\mathbf{1}=\mathbf{1}+3 \rho_{\left[2^{3}\right]}+3 \rho_{[42]}+\rho_{\left[31^{3}\right]}+\rho_{[321]}, \\
M_{8}\left(\Gamma_{3}(2)\right)=\mathbf{1}+4 \cdot \mathbf{1 5}_{a}+\mathbf{3 5}_{a}+4 \cdot \mathbf{3 5}_{b}+5 \cdot \mathbf{8 4}_{a}+2 \cdot \mathbf{1 0 5}_{c}+\mathbf{1 6 8}_{a}+ \\
2 \cdot \mathbf{1 8 9}_{c}+3 \cdot \mathbf{2 1 6}+3 \cdot \mathbf{2 8 0}_{b}+2 \cdot \mathbf{3 3 6}_{a}+\mathbf{4 2 0}_{a} .
\end{aligned}
$$

From the Frobenius identity it follows that for $g=1$ the dimension of the space of $O^{+}$-invariants is three, for $g=2$ is four and for $g=3$ is five. For genus four and five we do not know the decomposition of the whole $M_{8}\left(\Gamma_{g}(2)\right)$ and, moreover, the ring of modular forms is not understood in terms of Heisenberg invariant polynomials in theta constants. However, in Section [6 and 7, we will restrict our attention to the space $M_{8}^{\theta}\left(\Gamma_{g}(2)\right)$, searching the $O^{+}$-invariants there.

\section{The construction of the $\mathrm{O}^{+}$-invariants}

Once the dimension of $M_{8}\left(\Gamma_{g}(2)\right)^{O^{+}}$is known, the main problem is to find an explicit expression for a basis of this space in terms of theta constants, if possible. In [7] and 8. the notion of isotropic subspaces was employed to find such bases. Recall that, if $V$ is provided with a symplectic form, $W \subset V$ is an isotropic subspace if on every pair of vectors in $W$ the symplectic form vanishes. This way to determine the invariants makes use of the geometry underlying the theta characteristics and the corresponding action of the symplectic group on them. For example, the condition for a subspace to be isotropic is preserved under the action of $\mathrm{Sp}(2 g)$. Moreover, it is quite simple to determine the restriction on a block diagonal period matrix of the $\mathrm{O}^{+}$-invariant built in this way, despite to the huge number of terms appearing in these functions, cf. the discussion in Appendix $\mathrm{C}$ of [7. The knowledge of a basis for these spaces allows to find, for $g \leq 5$, a linear combination of the $\mathrm{O}^{+}$-invariants such that its restrictions fits all requests in the ansätze discussed in Section 2. For $g=1,2,3$ the fact that any modular form of weight $2 k$ can be expressed as a polynomial of degree $4 k$ in theta constants in a unique way (unique up to a multiple of $J^{(3)}$ if $g=3$ and $k>4$, see Section 21) allows us to prove the uniqueness for the expression of the superstring measure. In genus four the ring of Siegel modular forms is not normal. This means that in general there could be some modular forms 
that cannot be expressed as polynomials in theta constants. In this case in [10] the uniqueness was proved in a weakened form, assuming the polynomiality for the amplitude, i.e. considering $O^{+}$-invariants contained in the space $M_{8}^{\theta}\left(\Gamma_{4}(2)\right)$ only. In 27] the proof is also extended to the general case. As anticipated in Section 1, in genus five the three constraints are not strong enough to assure the uniqueness of the superstring measure neither if we restrict to the normal part of the ring of modular forms as we will prove in Section 7 and in [9]. Thus, in genus five the loss of the uniqueness is not due just to the non normality of the ring of modular forms.

In [17] a generalisation for the expression of the chiral measure at any genus $g$ was proposed. In the approach used there, the action of the symplectic group underlying that expression is not manifest although the correct factorisation is obtained. The author restricts the search for the $g$ loop amplitudes to a suitable vector space of dimension $g+1$ then finding there a unique solution of the constraints. However, for genus three (four and more) the vector space defined by the transformation constraint has dimension five $(\geq 7)$, see [10] 7.4 and 7.5, which is larger than the dimensions of the starting spaces selected in [17]. Moreover, his expression might be not well-defined for $g>5$ (Salvati Manni in [33] discusses the case $g=5$ ) due to the presence of some roots.

We will now provide new expressions for $\Xi_{8}^{(g)}\left[\Delta^{(g)}\right]$ at lower genus. In these new formulas, the theta constants appear at higher power than in the expressions given in all previous works.

\section{1 $\mathrm{O}^{+}$-invariants for the genus 3 case}

For genus $g=3$ the only $\operatorname{Sp}(6)$-representations that have an $O^{+}$-invariant are $\mathbf{1}$ and $\sigma_{\theta}=\mathbf{3 5} \mathbf{5}_{b}$ and we know that there are five such linearly independent invariants, see [10. The representation $\mathbf{1}$ provides the $\operatorname{Sp}(6)$-invariant and $\mathbf{3} \mathbf{5}_{b}$ the representation on the $\theta\left[\Delta^{(3)}\right]^{8}$. A natural question is about the number of linearly independent $\mathrm{O}^{+}$-invariants of degree 16 that can be written as quadratic polynomials in $\theta\left[\Delta^{(3)}\right]^{8}$. From the decomposition of the tensor products in irreducible representations we find that:

$$
\begin{aligned}
& \operatorname{Sym}^{2}\left(\mathbf{1}+\mathbf{3} \boldsymbol{5}_{b}\right)=\mathbf{1}+\mathbf{3} \boldsymbol{5}_{b}+\operatorname{Sym}^{2}\left(\mathbf{3} \boldsymbol{5}_{b}\right) \\
& =1+35_{b}+1+27_{a}+2 \cdot 35_{b}+84_{a}+168_{a}+280_{b}
\end{aligned}
$$

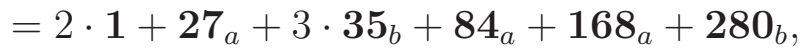

so that we get the two $\operatorname{Sp}(6)$-invariants $\sum_{\Delta} \theta\left[\Delta^{(3)}\right]^{16}$ and $\left(\sum_{\Delta} \theta\left[\Delta^{(3)}\right]^{8}\right)^{2}$ and three $O^{+}$-invariants (but not $\operatorname{Sp}(6)$ ), two of which are $\theta\left[0^{(3)}\right]^{16}$ and $\theta\left[0^{(3)}\right]^{8} \sum_{\Delta} \theta\left[\Delta^{(3)}\right]^{8}$. In 
order to find the third invariant quadratic in $\theta\left[\Delta^{(3)}\right]^{8}$ we can adopt a general method that allows us to generate many $\mathrm{O}^{+}$-invariants (clearly not all independent). This consists in starting from a certain monomial of degree sixteen which contains the theta constants to the power at least four, and imposing some suitable condition on the corresponding characteristics. For example, in the spirit of [12] and [13], we can take $\theta\left[\Delta_{1}^{(3)}\right]^{4} \theta\left[\Delta_{2}^{(3)}\right]^{4} \theta\left[\Delta_{3}^{(3)}\right]^{4} \theta\left[\Delta_{4}^{(3)}\right]^{4}$ with the conditions $\Delta_{1}+\Delta_{2}+\Delta_{3}+\Delta_{4}=0^{(3)}$. There are 1611 such monomials which summed up give an $O^{+}$-invariant. In fact, this is redundant because there are "sub-polynomials" that are orbits for $\mathrm{O}^{+}$and then are themselves invariant. In [10] Section 8.5, the generators of $O^{+} \cong S_{8}$ are given in terms of transvections acting on the theta constants. Thus, an orbit can be determined acting on a single monomial with these transvections until the number of terms of the generated polynomial stops to grow. Next, one considers a second monomial (not in the orbit of the first) and repeats the procedure. In this way we can recognise eight $\mathrm{O}^{+}$-invariants inside the big polynomial, as shown in Table 3. It is clear that the searched invariant could be the sixth or the seventh. Using a computer or (quite lengthy!) by hand and the classical theta formula (cf. [7], Section 3.2), we verify that each of them is linearly independent from the other invariants. We then choose the sixth, which we will call $F_{88}^{(3)}$ (and $F_{88}^{(g)}$ for arbitrary genus $g$ ). Thus, each monomial in $F_{88}^{(3)}$ is the product of two theta constants at the eighth power, with the conditions that the sum of their characteristics is odd (even, if we choose the seventh), the two characteristics are not equal and both are not zero. Note the following equality between the $\mathrm{O}^{+}$-invariants:

$$
\begin{aligned}
\left(\sum_{\Delta} \theta\left[\Delta^{(3)}\right]^{8}\right)^{2}-\sum_{\Delta} \theta\left[\Delta^{(3)}\right]^{16} & =2 \sum_{\left(\Delta_{i}, \Delta_{j}\right)_{e}} \theta\left[\Delta_{i}^{(3)}\right]^{8} \theta\left[\Delta_{j}^{(3)}\right]^{8}+2 \sum_{\left(\Delta_{i}, \Delta_{j}\right)_{o}} \theta\left[\Delta_{i}^{(3)}\right]^{8} \theta\left[\Delta_{j}^{(3)}\right]^{8} \\
& +2\left(\theta\left[0^{(g)}\right]^{8} \sum_{\Delta} \theta\left[\Delta^{(3)}\right]^{8}-\theta\left[0^{(3)}\right]^{16}\right)
\end{aligned}
$$

where the first two functions on the r.h.s. are the $\mathrm{O}^{+}$-invariants of lines six and seven of the Table 3 and the "e" and "o" stand for even sum and odd sum of the two characteristics respectively. The two functions on the l.h.s. are the two $\operatorname{Sp}(6)-$ invariants, $F_{8}^{(3)}$ and $F_{16}^{(3)}$ as we will call them in the following. In fact, for genus three the two $\mathrm{Sp}(6)$-invariants are not linearly independent but there is a relation between them (the $J^{(3)}$, see below, or $F_{16}$ in the notation of [10]). Therefore, to find a basis we need to look for another invariant which cannot be expressed as a quadratic polynomial in $\theta\left[\Delta^{(3)}\right]^{8}$. We can take $\theta\left[0^{(3)}\right]^{4} \sum_{\Delta} \theta\left[\Delta^{(3)}\right]^{12}$.

In Section 5 we will show how to build the chiral measure from these functions. 


\begin{tabular}{|c|c|c|c|}
\hline orbit & general expression & condition & num. elem. \\
\hline 1 & $\theta[0]^{16}$ & & 1 \\
\hline 2 & $\theta[\Delta]^{16}$ & $\Delta \neq 0$ & 35 \\
\hline 3 & $\theta[0]^{8} \sum_{\Delta} \theta[\Delta]^{8}$ & $\Delta \neq 0$ & 35 \\
\hline 4 & $\theta[0]^{4} \sum_{\Delta_{i}, \Delta_{j}, \Delta_{k}} \theta\left[\Delta_{i}\right]^{4} \theta\left[\Delta_{j}\right]^{4} \theta\left[\Delta_{k}\right]^{4}$ & $\begin{array}{l}\Delta_{i}+\Delta_{j}+\Delta_{k}=0 \\
\Delta_{i}, \Delta_{j}, \Delta_{k} \neq 0 \\
\Delta_{i} \neq \Delta_{j} \neq \Delta_{k}\end{array}$ & 105 \\
\hline 5 & $\sum_{\Delta_{i}, \Delta_{j}, \Delta_{k}, \Delta_{l}} \theta\left[\Delta_{i}\right]^{4} \theta\left[\Delta_{j}\right]^{4} \theta\left[\Delta_{k}\right]^{4} \theta\left[\Delta_{l}\right]^{4}$ & $\begin{array}{l}\Delta_{i}+\Delta_{j}+\Delta_{k}+\Delta_{l}=0 \\
\Delta_{i}+\Delta_{j}+\Delta_{k} \text { even } \\
\Delta_{i}+\Delta_{j} \text { even } \\
\Delta_{i}, \Delta_{j}, \Delta_{k}, \Delta_{l} \neq 0 \\
\Delta_{i} \neq \Delta_{j} \neq \Delta_{k} \neq \Delta_{l}\end{array}$ & 210 \\
\hline 6 & $\sum_{\Delta_{i}, \Delta_{j}} \theta\left[\Delta_{i}\right]^{8} \theta\left[\Delta_{j}\right]^{8}$ & $\begin{array}{l}\Delta_{i}+\Delta_{j} \text { odd } \\
\Delta_{i}, \Delta_{j} \neq 0 \\
\Delta_{i} \neq \Delta_{j}\end{array}$ & 280 \\
\hline 7 & $\sum_{\Delta_{i}, \Delta_{j}} \theta\left[\Delta_{i}\right]^{8} \theta\left[\Delta_{j}\right]^{8}$ & $\begin{array}{l}\Delta_{i}+\Delta_{j} \text { even } \\
\Delta_{i}, \Delta_{j} \neq 0 \\
\Delta_{i} \neq \Delta_{j}\end{array}$ & 315 \\
\hline 8 & $\sum_{\Delta_{i}, \Delta_{j}, \Delta_{k}, \Delta_{l}} \theta\left[\Delta_{i}\right]^{4} \theta\left[\Delta_{j}\right]^{4} \theta\left[\Delta_{k}\right]^{4} \theta\left[\Delta_{l}\right]^{4}$ & $\begin{array}{l}\Delta_{i}+\Delta_{j}+\Delta_{k}+\Delta_{l}=0 \\
\Delta_{i}+\Delta_{j}+\Delta_{k} \text { even } \\
\Delta_{i}+\Delta_{j} \text { even } \\
\Delta_{k}+\Delta_{l} \text { odd } \\
\Delta_{i}, \Delta_{j}, \Delta_{k}, \Delta_{l} \neq 0 \\
\Delta_{i} \neq \Delta_{j} \neq \Delta_{k} \neq \Delta_{l}\end{array}$ & 630 \\
\hline
\end{tabular}

Table 3

Orbits under the action of $O^{+}$(genus three case).

3.2 $\mathrm{O}^{+}$-invariants for the genus 4 case.

For genus $g=4$, the only $\operatorname{Sp}(8)$-representations containing an $O^{+}$-invariant are 1 and 135. Now, it is not known if $M_{2 k}^{\theta}\left(\Gamma_{4}(2)\right)$, the space of modular forms of weight $2 k$ which are (Heisenberg-invariants) polynomial in $\Theta[\sigma]$ 's, coincides with $M_{2 k}\left(\Gamma_{4}(2)\right)$. Recently, Oura determined the dimension of $M_{2 k}^{\theta}\left(\Gamma_{g}(2)\right)^{O^{+}}$obtaining 7 for the $g=4$ case. In principle these dimensions could also be computed using a method similar to those we used for $g<4$, i.e. searching for the decomposition of $M_{8}^{\theta}\left(\Gamma_{g}(2)\right)$ in irreducible representations, but it is very time and memory consuming 
for increasing $g$. As for the genus three case, we want to find a basis for the $\mathrm{O}^{+}$invariants in which the theta constants appear with the highest possible degree. Let us start by determining the decomposition of the symmetric product $\operatorname{Sym}^{2}(\mathbf{1}+\mathbf{1 3 5})$ in irreducible representations. This can be done using Magma or, by hand, with the character table of $\operatorname{Sp}(8)$ and the character inner product (see [6], Section 5.2.1, for the case $g=2$ ). We obtain the decomposition:

$$
\begin{aligned}
\operatorname{Sym}^{2}(1+135) & =\mathbf{1}+\mathbf{1 3 5}+\operatorname{Sym}^{2}(\mathbf{1 3 5}) \\
& =2 \cdot \mathbf{1}+\mathbf{1 1 9}+3 \cdot \mathbf{1 3 5}+\mathbf{1 1 9 0}+\mathbf{3 4 0 0}+\mathbf{4 2 0 0} .
\end{aligned}
$$

This means that we can find five $O^{+}$-invariants that are quadratic polynomials in the theta constants at the eighth power. Two of them are the $\mathrm{Sp}(8)$-invariants $\sum_{\Delta} \theta\left[\Delta^{(4)}\right]^{16}$ and $\left(\sum_{\Delta} \theta\left[\Delta^{(4)}\right]^{8}\right)^{2}$ which are now linearly independent because the Schottky relation $J^{(4)}$, the analogous of $J^{(3)}$ for genus four (see Section 3.4 for the definition), vanishes just on $J_{4}$ and not identically on the whole $\mathbf{H}_{4}$. The remaining three invariants are $\theta\left[0^{(4)}\right]^{16}, \theta\left[0^{(4)}\right]^{8} \sum_{\Delta} \theta\left[\Delta^{(4)}\right]^{8}$ and the generalisation of the $O^{+}$ invariant found in Section 3.1 to the genus four case, $F_{88}^{(4)}$ (the construction of such a function for $g \geq 4$ is straightforward).

We now check how many $\mathrm{O}^{+}$-invariants can be written as polynomials of degree four in the $\theta\left[\Delta^{(4)}\right]^{4}$. This can be done decomposing the symmetric product $\operatorname{Sym}^{4}\left(\rho_{\theta}\right)$ in irreducible representation 4 , and counting the multiplicity of the representations 1 and $\sigma_{\theta}\left(\sigma_{\theta}=135\right.$ in this case). In [35] it was shown that the representation of $\operatorname{Sp}(2 g)$ on the subspace $M_{2}^{\theta}\left(\Gamma_{g}(2)\right) \subset M_{2}\left(\Gamma_{g}(2)\right)$, that is spanned by the $\theta\left[\Delta^{(g)}\right]^{4}$, is isomorphic to the representation $\rho_{\theta}$ found by Frame [16] that supports $O^{+}$-antiinvariants. This representation has dimension $\operatorname{dim} \rho_{\theta}=\left(2^{g}+1\right)\left(2^{g-1}+1\right) / 3$, so for $g=4$ one finds $\rho_{\theta}=51$; see [10] for details. Thus, a function belonging to $\operatorname{Sym}^{2 n}\left(\rho_{\theta}\right)$ is an $O^{+}$-invariant of degree $2 n$ in $\theta\left[\Delta^{(g)}\right]^{4}, n \in \mathbf{N}$. We have:

$$
\begin{aligned}
& \operatorname{Sym}^{4}(\mathbf{5 1})=2 \cdot \mathbf{1}+\mathbf{5 1}+\mathbf{1 1 9}+4 \cdot \mathbf{1 3 5}+\mathbf{5 1 0}+2 \cdot \mathbf{9 1 8}+5 \cdot \mathbf{1 1 9 0}+\mathbf{1 2 7 5} \\
& +2856_{-504}+2 \cdot 3400+3 \cdot 4200+5712+5950_{-210}+7140 \\
& +8160+\mathbf{1 1 9 0 0}_{700}+3 \cdot \mathbf{1 3 6 0 0}+\mathbf{1 8 3 6 0}+2 \cdot \mathbf{1 9 0 4 0}+\mathbf{2 3 8 0 0 _ { - 1 9 6 0 }} \\
& +32130_{2898}+34560+57120 \text {, }
\end{aligned}
$$

so we get six $O^{+}$-invariants, the five found before and $\theta\left[0^{(4)}\right]^{4} \sum_{\Delta} \theta\left[\Delta^{(4)}\right]^{12}$. The seventh invariant cannot be written in this way, but we can search for it as a polynomial in the $\theta\left[\Delta^{(2)}\right]^{2}$. In general, we have not a representation of $\operatorname{Sp}(2 g)$ on the space generated by the $\theta\left[\Delta^{(g)}\right]^{2}$. So we cannot repeat the previous method using something like $\operatorname{Sym}^{8}(\cdots)$. However, we already know at least one $O^{+}$-invariant linearly independent from the others that can be written as a polynomial in $\theta\left[\Delta^{(4)}\right]^{2}$ : the invariant

$\overline{4 \text { Here }} \rho_{\theta}$ is the representation of $\operatorname{Sp}(2 g)$ on the $\theta\left[\Delta^{(g)}\right]^{4}(2 g=8$ in this case). 
$G_{1}\left[0^{(4)}\right]$ defined in $\left[8\right.$, which, for later convenience, will be renamed $5 G_{3}^{(4)}\left[0^{(4)}\right]$. Using a computer, we have verified that it is linearly independent from the other six. The same conclusion can be achieved using the approach of [9]. Let us recall the definition of the function $G_{3}^{(4)}\left[0^{(4)}\right]$, or $P_{3,2}^{(4)}$ in Grushevsky notation [17]. Given any three dimensional isotropic subspace $W \in \mathbf{F}_{2}^{8}$, there are $3 \cdot 8=24$ even quadrics $Q_{\Delta}$ such that $W \subset Q_{\Delta}$. Let $Q_{0} \subset \mathbf{F}_{2}^{8}$ be the even quadric with characteristic $\Delta_{0}^{(4)}=\left[0^{(4)}\right]$. We will use only the octets of quadrics which contain $Q_{0}$ to define a modular form $G_{3}^{(4)}\left[0^{(4)}\right]$ :

$$
G_{3}^{(4)}\left[0^{(4)}\right]=\sum_{W \subset Q_{0}} \prod_{w \in W} \theta\left[\Delta_{0}^{(4)}+w\right]^{2},
$$

where we sum over the 2025 three dimensional isotropic subspaces $W \subset Q_{0}$, and for each such subspace we take the product of the eight even $\theta\left[\Delta_{0}^{(4)}+w\right]^{2}$. As explained in [8] it is a modular form on $\Gamma_{4}(1,2)$.

Having found seven linearly independent $\mathrm{O}^{+}$-invariants, according to Oura's result, we have a basis for $M_{8}^{\theta}\left(\Gamma_{4}(2)\right)^{O^{+}}$and in Section 6 we will search for a linear combination of them to build the function $\Xi_{8}^{(4)}\left[0^{(4)}\right]$ which restricts correctly.

\section{3 $\mathrm{O}^{+}$-invariants for the genus 5 case}

For genus five, the ring of modular forms, as for $g=4$, is not normal. Moreover, there may exist many relations satisfied by the second order theta constants, but in any case they are not known. Finally, the Schottky relation does not vanish on the Jacobi locus (although this was conjectured by Belavin and Knizhnik [3, Conjecture 3, by Morozov and Perelemov [4,25] and by D'Hoker and Phong in [13], Section 4.1 and it was shown that it vanishes for any genus on the hyperelliptic locus by Poor [28]): a very recent result [18], Corollary 18, shows that the zero locus of this form is the locus of trigonal curves.

Despite these difficulties, starting from the seven functions and mimicking the $g=4$ invariants, we can try to add a further linearly independent $O^{+}$-invariant polynomial and look for a linear combination (possibly unique) which factorises in the right way. Indeed, for genus five it is known that

$$
\operatorname{dim} M_{8}^{\theta^{2}}\left(\Gamma_{5}(2)\right)^{O^{+}} \leq \operatorname{dim} M_{8}^{\theta}\left(\Gamma_{5}(2)\right)^{O^{+}} \leq \operatorname{dim} M_{8}^{\theta_{S}}\left(\Gamma_{5}(2)\right)^{O^{+}}=8,
$$

5 We made a change of notation with respect our previous works: all the forms built using the isotropic space will be indicated by $G_{d}^{(g)}\left[0^{(g)}\right]$, where $d$ is the dimension of the isotropic subspace and $g$ the genus we are considering. For example the form $H\left[0^{(3)}\right]$ of 8 ] becomes $G_{2}^{(3)}\left[0^{(3)}\right]$ in the new notation. 
where the first term is the space of modular forms with respect to the group $\Gamma_{5}(1,2)$ of weight eight which are polynomial in $\theta\left[\Delta^{(5)}\right]^{2}$, the second is the space of modular forms polynomial in $\theta\left[\Delta^{(5)}\right]$ (w.r.t. the same group and of same weight as before), and the third is the space of the theta series associated to quadratic forms, see [1]. Note that it is not clear that $M_{8}^{\theta}\left(\Gamma_{5}(2)\right)^{O^{+}}$is a subset of $M_{8}^{\theta_{S}}\left(\Gamma_{5}(2)\right)^{O^{+}}$, in fact in paper [9] we have shown that these two spaces are the same.

In order to construct a basis for $M_{8}^{\theta}\left(\Gamma_{5}(2)\right)^{O^{+}}$we generalise the form $G_{3}^{(4)}\left[0^{(4)}\right]$ used before and the inequalities (2) show that we can find at most one $\mathrm{O}^{+}$-invariant polynomial in $\theta\left[\Delta^{(5)}\right]$. To this aim we consider the form $G_{4}^{(4)}\left[0^{(4)}\right]$ introduced in [8] and define it also for the $g=5$ case. This goes straightforward with the notion of isotropic subspaces and in principle we can define similar forms for arbitrary genus $g$ using isotropic subspaces of dimension at most $g$ (see e.g. [17]).

\subsubsection{The form $G_{3}^{(5)}\left[0^{(5)}\right]$}

We will follow the definitions of [8]. Let $W \subset \mathbf{F}_{2}^{10}$ be a three dimensional isotropic subspace. Given such a $W$, there are $10 \cdot 8=80$ even quadrics $Q_{\Delta}$ such that $W \subset Q_{\Delta}$. Let $Q_{0} \subset \mathbf{F}_{2}^{10}$ be the even quadric with characteristic $\Delta_{0}^{(5)}=\left[0^{(5)}\right]$. We will only use the octets of quadrics which contain $Q_{0}$ to define the modular form $G_{3}^{(5)}\left[0^{(5)}\right]$, or $P_{3,2}^{(5)}$ in the notations of [17]:

$$
G_{3}^{(5)}\left[0^{(5)}\right]:=\sum_{W \subset Q_{0}} \prod_{w \in W} \theta\left[\Delta_{0}^{(5)}+w\right]^{2}
$$

where we sum over the 118575 three dimensional isotropic subspaces $W \subset Q_{0}$, and for each such subspace we take the product of the eight $\theta\left[\Delta_{0}^{(5)}+w\right]^{2}$.

\subsubsection{The form $G_{4}^{(5)}\left[0^{(5)}\right]$}

Let $W \subset \mathbf{F}_{2}^{10}$ be a four dimensional isotropic subspace. Given such a $W$, there are 48 even quadrics $Q_{\Delta}$ such that $W \subset Q_{\Delta}$. Let $Q_{0} \subset \mathbf{F}_{2}^{10}$ be the even quadric with characteristic $\Delta_{0}^{(5)}=\left[0^{(5)}\right]$. We will only use the sets of quadrics which contain $Q_{0}$ to define the modular form $G_{4}^{(5)}\left[0^{(5)}\right]$, or $P_{4,1}^{(5)}$ as in [17]:

$$
G_{4}^{(5)}\left[0^{(5)}\right]:=\sum_{W \subset Q_{0}} \prod_{w \in W} \theta\left[\Delta_{0}^{(5)}+w\right]
$$

where we sum over the 71145 four dimensional isotropic subspaces $W \subset Q_{0}$, and for each such subspace we take the product of the sixteen $\theta\left[\Delta_{0}^{(5)}+w\right]$. 


\subsubsection{Remark}

The eight functions $F_{1}^{(5)}, F_{2}^{(5)}, F_{3}^{(5)}, F_{8}^{(5)}, F_{18}^{(5)}, F_{16}^{(5)}, G_{3}^{(5)}\left[0^{(5)}\right], G_{4}^{(5)}\left[0^{(5)}\right]$ are linearly independent, as can be checked by a computer or by the restriction we will deduce in Section 7 or using the technique of [9]. Thus, it follows that in (2) an equality must hold between the second and the third term:

$$
\operatorname{dim} M_{8}^{\theta}\left(\Gamma_{5}(2)\right)^{O^{+}}=\operatorname{dim} M_{8}^{\theta_{S}}\left(\Gamma_{5}(2)\right)^{O^{+}}=8
$$

From the computations at genus four, given in Section [6, and from a result of Nebe [26], it also follows that at genus five:

$$
\operatorname{dim} M_{8}^{\theta^{2}}\left(\Gamma_{5}(2)\right)^{O^{+}}=7
$$

Indeed, we obtain seven linear independent functions in the space $M_{8}^{\theta^{2}}\left(\Gamma_{5}(2)\right)^{O^{+}}$ so its dimension is greater or equal than seven. In [26] it was determined seven as an upper limit for the dimension of the space of theta square series associated to quadratic forms. As this space contains $M_{8}^{\theta^{2}}\left(\Gamma_{5}(2)\right)^{O^{+}}$, it follows the equality (3). This result can also be checked using the method of [9]. This fixes all the dimensions of the spaces appearing in the previous inequality (2).

\subsection{Genus $g$ expressions for $\mathrm{O}^{+}$-invariants}

In this Section we recall the six $O^{+}$-invariants belonging to $\mathrm{Sym}^{4} \rho_{\theta}$, found for the lower genus, and we generalise them for arbitrary $g$. The first three are the same as in [7], but multiplied by $\theta\left[0^{(g)}\right]^{4}$ to get a form of weight eight; $F_{8}^{(g)}$ and $F_{16}^{(g)}$ are the two $\operatorname{Sp}(2 g)$-invariants, $F_{88}^{(g)}$ is the generalised $O^{+}$-invariant introduced in Section 3.1 and the modular form $J^{(g)}:=2^{g} F_{16}^{(g)}-F_{8}^{(g)}$ vanishes identically for genus three, as 
explained in [7]. These are:

$$
\begin{aligned}
& F_{1}^{(g)}:=\theta\left[0^{(g)}\right]^{16}, \\
& F_{2}^{(g)}:=\theta\left[0^{(g)}\right]^{4} \sum_{\Delta^{(g)}} \theta\left[\Delta^{(g)}\right]^{12}, \\
& F_{3}^{(g)}:=\theta\left[0^{(g)}\right]^{8} \sum_{\Delta^{(g)}} \theta\left[\Delta^{(g)}\right]^{8}, \\
& F_{8}^{(g)}:=\left(\sum_{\Delta^{(g)}} \theta\left[\Delta^{(g)}\right]^{8}\right)^{2}, \\
& F_{88}^{(g)}:=\sum_{\left(\Delta_{i}^{(g)}, \Delta_{j}^{(g)}\right)_{o}} \theta\left[\Delta_{i}^{(g)}\right]^{8} \theta\left[\Delta_{j}^{(g)}\right]^{8}, \\
& F_{16}^{(g)}:=\sum_{\Delta^{(g)}} \theta\left[\Delta^{(g)}\right]^{16}, \\
& J^{(g)}:=2^{g} \sum_{\Delta^{(g)}} \theta\left[\Delta^{(g)}\right]^{16}-\left(\sum_{\Delta^{(g)}} \theta\left[\Delta^{(g)}\right]^{8}\right)^{2}=2^{g} F_{16}^{(g)}-F_{8}^{(g)},
\end{aligned}
$$

where $\left(\Delta_{i}^{(g)}, \Delta_{j}^{(g)}\right)_{o}$ stands for all the pairs of distinct even characteristics such that their sum is odd. Behind these, we also introduced the forms $G_{3}^{(g)}\left[0^{(g)}\right]$ for $g=4,5$ and $G_{4}^{(g)}\left[0^{(g)}\right]$ for $g=5$. However $G_{3}^{(g)}\left[0^{(g)}\right]\left(G_{4}^{(g)}\left[0^{(g)}\right]\right)$ could be defined for every genu $6 \geq 3(g \geq 4)$ considering three (four) dimensional isotropic subspace of $\mathbf{F}_{2}^{2 g}$. In the same way, we can consider two dimensional isotropic subspaces of $\mathbf{F}_{2}^{2 g}$, for $g \geq 2$ and introduce another $\mathrm{O}^{+}$-invariant, $G_{2}^{(g)}\left[0^{(g)}\right]$ (clearly not linear independent from the others), that is a sum of suitable products of four theta constants at the fourth power. This form will appear in the factorisation of some $\mathrm{O}^{+}$-invariants.

\section{Factorization of the $\mathrm{O}^{+}$-invariants}

\subsection{Genus one formulae}

For the construction of the forms $\Xi_{8}^{(g)}\left[0^{(g)}\right]$ defining the chiral measure and to check that they have the correct restriction on $\mathbf{H}_{1} \times \mathbf{H}_{g-1}$, it will be useful to recall some identities between theta constants at genus one. We will use the Dedekind function $\eta$ for which the classical formula $\eta^{3}=\theta\left[\begin{array}{l}0 \\ 0\end{array}\right] \theta\left[\begin{array}{l}0 \\ 1\end{array}\right] \theta\left[\begin{array}{l}1 \\ 0\end{array}\right]$ holds 4 , so $3 \eta^{12}=\theta\left[\begin{array}{l}0 \\ 0\end{array}\right]^{12}-\theta\left[\begin{array}{l}0 \\ 1\end{array}\right]^{12}-\theta\left[\begin{array}{l}1 \\ 0\end{array}\right]^{12}$. Also we consider the function $f_{21}=2 \theta\left[\begin{array}{l}0 \\ 0\end{array}\right]^{12}+\theta\left[\begin{array}{l}0 \\ 1\end{array}\right]^{12}+\theta\left[\begin{array}{l}1 \\ 0\end{array}\right]^{12}$ as in [7]. The two functions

$\overline{6}$ In [7], where it was considered the case $g=3$, this form is called $G[0]$.

7 Note that our definition of the Dedekind function differs from the classical ones, cf. [29], for a factor $\frac{1}{2}$. This explains the difference for a global factor $2^{4 g}$ between our definition 
$\eta^{12}$ and $f_{21}$ are a basis for the genus one $O^{+}$-anti-invariants [10] and so we can expand the anti-invariants on this basis and the $O^{+}$-invariants on the basis $\theta\left[\begin{array}{l}0 \\ 0\end{array}\right]^{4} \eta^{21}, \theta\left[\begin{array}{l}0 \\ 0\end{array}\right]^{4} f_{21}$ and $F_{16}^{(1)}=\theta\left[\begin{array}{l}0 \\ 0\end{array}\right]^{16}+\theta\left[\begin{array}{l}1 \\ 0\end{array}\right]^{16}+\theta\left[\begin{array}{l}0 \\ 1\end{array}\right]^{16}$. The proof of these identities is straightforward using the Jacobi identity $\theta\left[\begin{array}{l}0 \\ 0\end{array}\right]^{4}=\theta\left[\begin{array}{l}0 \\ 1\end{array}\right]^{4}+\theta\left[\begin{array}{l}1 \\ 0\end{array}\right]^{4}$ :

$$
\begin{aligned}
& \theta^{12}\left[\begin{array}{l}
0 \\
0
\end{array}\right]=\frac{1}{3} f_{21}+\eta^{12}, \\
& \theta\left[\begin{array}{l}
0 \\
0
\end{array}\right]^{4}\left(\theta\left[\begin{array}{l}
0 \\
0
\end{array}\right]^{8}+\theta\left[\begin{array}{l}
0 \\
1
\end{array}\right]^{8}+\theta\left[\begin{array}{l}
1 \\
0
\end{array}\right]^{8}\right)=\frac{2}{3} f_{21} \\
& \theta\left[\begin{array}{l}
0 \\
0
\end{array}\right]^{12}+\theta\left[\begin{array}{l}
0 \\
1
\end{array}\right]^{12}+\theta\left[\begin{array}{l}
1 \\
0
\end{array}\right]^{12}=\frac{2}{3} f_{21}-\eta^{12}, \quad \theta\left[\begin{array}{l}
0 \\
0
\end{array}\right]^{4} \theta\left[\begin{array}{l}
0 \\
1
\end{array}\right]^{8}+\theta\left[\begin{array}{l}
0 \\
0
\end{array}\right]^{4} \theta\left[\begin{array}{l}
1 \\
0
\end{array}\right]^{8}=\frac{1}{3} f_{21}-\eta^{12}, \\
& \theta\left[\begin{array}{l}
0 \\
0
\end{array}\right]^{16}+2 \theta\left[\begin{array}{l}
0 \\
0
\end{array}\right]^{8} \theta\left[\begin{array}{l}
0 \\
1
\end{array}\right]^{8}+2 \theta\left[\begin{array}{l}
0 \\
0
\end{array}\right]^{8} \theta\left[\begin{array}{l}
1 \\
0
\end{array}\right]^{8}=\theta\left[\begin{array}{l}
0 \\
0
\end{array}\right]^{4}\left(f_{21}-\eta^{12}\right) \\
& \frac{1}{2} \theta\left[\begin{array}{l}
0 \\
0
\end{array}\right]^{8} \theta\left[\begin{array}{l}
0 \\
1
\end{array}\right]^{8}+\frac{1}{2} \theta\left[\begin{array}{l}
0 \\
0
\end{array}\right]^{8} \theta\left[\begin{array}{l}
1 \\
0
\end{array}\right]^{8}-\theta\left[\begin{array}{l}
0 \\
0
\end{array}\right]^{4} \theta\left[\begin{array}{l}
0 \\
1
\end{array}\right]^{12}-\theta\left[\begin{array}{l}
0 \\
0
\end{array}\right]^{4} \theta\left[\begin{array}{l}
1 \\
0
\end{array}\right]^{12}=\theta\left[\begin{array}{l}
0 \\
0
\end{array}\right]^{4}\left(\frac{3}{2} \eta^{12}-\frac{1}{6} f_{21}\right), \\
& \theta\left[\begin{array}{l}
0 \\
0
\end{array}\right]^{16}+3 \theta\left[\begin{array}{l}
0 \\
0
\end{array}\right]^{8} \theta\left[\begin{array}{l}
0 \\
1
\end{array}\right]^{8}+3 \theta\left[\begin{array}{l}
0 \\
0
\end{array}\right]^{8} \theta\left[\begin{array}{l}
1 \\
0
\end{array}\right]^{8}-2 \theta\left[\begin{array}{l}
0 \\
0
\end{array}\right]^{4} \theta\left[\begin{array}{l}
0 \\
1
\end{array}\right]^{12}-2 \theta\left[\begin{array}{l}
0 \\
0
\end{array}\right]^{4} \theta\left[\begin{array}{l}
1 \\
0
\end{array}\right]^{12}=\theta\left[\begin{array}{l}
0 \\
0
\end{array}\right]^{4}\left(\frac{2}{3} f_{21}+2 \eta^{12}\right), \\
& \theta\left[\begin{array}{l}
0 \\
1
\end{array}\right]^{8} \theta\left[\begin{array}{l}
1 \\
0
\end{array}\right]^{8}=\frac{1}{2} \theta\left[\begin{array}{l}
0 \\
1
\end{array}\right]^{16}+\frac{1}{2} \theta\left[\begin{array}{l}
1 \\
0
\end{array}\right]^{16}+\frac{1}{2} \theta\left[\begin{array}{l}
0 \\
0
\end{array}\right]^{8} \theta\left[\begin{array}{l}
0 \\
1
\end{array}\right]^{8}+\frac{1}{2} \theta\left[\begin{array}{l}
0 \\
0
\end{array}\right]^{8} \theta\left[\begin{array}{l}
1 \\
0
\end{array}\right]^{8}-\theta\left[\begin{array}{l}
0 \\
0
\end{array}\right]^{4} \theta\left[\begin{array}{l}
0 \\
1
\end{array}\right]^{12}-\theta\left[\begin{array}{l}
0 \\
0
\end{array}\right]^{4} \theta\left[\begin{array}{l}
1 \\
0
\end{array}\right]^{12} \\
& =\frac{1}{2} F_{16}^{(1)}+\theta\left[\begin{array}{l}
0 \\
0
\end{array}\right]^{4}\left(\eta^{12}-\frac{1}{3} f_{21}\right) \text {. }
\end{aligned}
$$

\subsection{The restrictions on $\mathbf{H}_{1} \times \mathbf{H}_{g-1}$}

Let us report here the factorisation of the six $\mathrm{O}^{+}$-invariants found before for a reducible period matrix:

$$
\begin{aligned}
& F_{1 \mid \Delta_{1, g-1}}^{(g)}= \theta\left[\begin{array}{l}
0 \\
0
\end{array}\right]^{4}\left(\frac{1}{3} f_{21}+\eta^{12}\right) F_{1}^{(g-1)}, \\
& F_{2 \mid \Delta_{1, g-1}}^{(g)}= \theta\left[\begin{array}{l}
0 \\
0
\end{array}\right]^{4}\left(\frac{2}{3} f_{21}-\eta^{12}\right) F_{2}^{(g-1)}, \\
& F_{3 \mid \Delta_{1, g-1}}^{(g)}= \frac{2}{3} \theta\left[\begin{array}{l}
0 \\
0
\end{array}\right]^{4} f_{21} F_{3}^{(g-1)}, \\
& F_{8 \mid \Delta_{1, g-1}}^{(g)}=\left(\theta\left[\begin{array}{l}
0 \\
0
\end{array}\right]^{16}+\theta\left[\begin{array}{l}
0 \\
1
\end{array}\right]^{16}+\theta\left[\begin{array}{l}
1 \\
0
\end{array}\right]^{16}+2 \theta\left[\begin{array}{l}
0 \\
0
\end{array}\right]^{8} \theta\left[\begin{array}{l}
0 \\
1
\end{array}\right]^{8}+2 \theta\left[\begin{array}{l}
0 \\
0
\end{array}\right]^{8} \theta\left[\begin{array}{l}
1 \\
0
\end{array}\right]^{8}+2 \theta\left[\begin{array}{l}
0 \\
1
\end{array}\right]^{8} \theta\left[\begin{array}{l}
1 \\
0
\end{array}\right]^{8}\right) F_{8}^{(g-1)} \\
&= 2 F_{16}^{(1)} F_{8}^{(g-1)} \\
& F_{88 \mid \Delta_{1, g-1}}^{(g)}=\left(\theta\left[\begin{array}{l}
0 \\
0
\end{array}\right]^{16}+\theta\left[\begin{array}{l}
0 \\
1
\end{array}\right]^{16}+\theta\left[\begin{array}{l}
1 \\
0
\end{array}\right]^{16}+2 \theta\left[\begin{array}{l}
0 \\
0
\end{array}\right]^{8} \theta\left[\begin{array}{l}
0 \\
1
\end{array}\right]^{8}+2 \theta\left[\begin{array}{l}
0 \\
0
\end{array}\right]^{8} \theta\left[\begin{array}{l}
1 \\
0
\end{array}\right]^{8}-2 \theta\left[\begin{array}{l}
0 \\
1
\end{array}\right]^{8} \theta\left[\begin{array}{l}
1 \\
0
\end{array}\right]^{8}\right) F_{88}^{(g-1)} \\
&+\theta\left[\begin{array}{l}
0 \\
1
\end{array}\right]^{8} \theta\left[\begin{array}{l}
1 \\
0
\end{array}\right]^{8} F_{8}^{(g-1)} \\
&= \theta\left[\begin{array}{l}
0 \\
1
\end{array}\right]^{4} f_{21}\left(\frac{4}{3} F_{88}^{(g-1)}-\frac{1}{3} F_{8}^{(g-1)}\right)+\theta\left[\begin{array}{l}
0 \\
1
\end{array}\right]^{4} \eta^{12}\left(-4 F_{88}^{(g-1)}+F_{8}^{(g-1)}\right)+\frac{1}{2} F_{16}^{(1)} F_{8}^{(g-1)}, \\
&=\left(\theta\left[\begin{array}{l}
0 \\
0
\end{array}\right]^{16}+\theta\left[\begin{array}{l}
0 \\
1
\end{array}\right]^{16}+\theta\left[\begin{array}{l}
1 \\
0
\end{array}\right]^{16}\right) F_{16}^{(g-1)}=F_{16}^{(1)} F_{16}^{(g-1)} . \\
& F_{16 \mid \Delta_{1, g-1}}^{(g)}
\end{aligned}
$$

of the forms $\Xi_{8}^{(g)}\left[0^{(g)}\right]$ and the ones in [27]. 
The factorisation of the forms $G_{3}^{(g)}\left[0^{(g)}\right]$ and $G_{4}^{(g)}\left[0^{(g)}\right]$ can be determined for any $g$ using Theorem 15 of [17] and we report the result for the cases $g=4,5$ in Section 6 and 7 respectively.

\section{Genus three case}

In Section 3.1 we found a basis for the five dimensional space of $O^{+}$-invariants satisfying the transformation constraints. Let us search a linear combination satisfying the factorisation constraints. We will follow the strategy of [7]: write the more general vector in this space,

$$
\Xi_{8}^{(3)}\left[0^{(3)}\right]=a_{1} F_{1}^{(3)}+a_{2} F_{2}^{(3)}+a_{3} F_{3}^{(3)}+a_{4} F_{8}^{(3)}+a_{5} F_{88}^{(3)},
$$

and then impose for it to factorise as the product of the genus one form $\Xi_{8}^{(1)}\left[0^{(1)}\right]=$ $\theta\left[\begin{array}{l}0 \\ 0\end{array}\right]^{4} \eta^{12}$ and the form $\Xi_{8}^{(2)}\left[0^{(2)}\right]=\frac{2}{3} F_{1}^{(2)}+\frac{1}{3} F_{2}^{(2)}-\frac{1}{2} F_{3}^{(2)}$ at genus two, see [7], Section 3.4. In this way we obtain a linear equation in the five coefficients $a_{i}$.

\subsection{The restriction of $\Xi_{8}^{(3)}\left[0^{(3)}\right]$ on $\mathbf{H}_{1} \times \mathbf{H}_{2}$}

The factorisation of the expression (4) for a reducible period matrix of the form $\tau_{1,2}=\left(\begin{array}{cc}\tau_{1} & 0 \\ 0 & \tau_{2}\end{array}\right)$ is:

$$
\begin{aligned}
& \left(a_{1} F_{1}^{(3)}+a_{2} F_{2}^{(3)}+a_{3} F_{3}^{(3)}+a_{4} F_{8}^{(3)}+a_{5} F_{88}^{(3)}\right)\left(\tau_{1,2}\right) \\
& =\theta\left[\begin{array}{l}
0 \\
0
\end{array}\right]^{4}\left(a_{1}\left(\frac{1}{3} f_{21}+\eta^{12}\right) F_{1}^{(2)}+a_{2}\left(\frac{2}{3} f_{21}-\eta^{12}\right) F_{2}^{(2)}+a_{3} \frac{2}{3} f_{21} F_{3}^{(2)}\right) \\
& +a_{4} 2 F_{16}^{(1)} F_{8}^{(2)}+a_{5}\left[\theta\left[\begin{array}{l}
0 \\
1
\end{array}\right]^{4} f_{21}\left(\frac{4}{3} F_{88}^{(2)}-\frac{1}{3} F_{8}^{(2)}\right)+\theta\left[\begin{array}{l}
0 \\
1
\end{array}\right]^{4} \eta^{12}\left(-4 F_{88}^{(2)}+F_{8}^{(2)}\right)+\frac{1}{2} F_{16}^{(1)} F_{8}^{(2)}\right]
\end{aligned}
$$

Necessary condition for the restriction to take the form:

$$
\begin{aligned}
& \left(\Xi_{8}^{(3)}\left[0^{(3)}\right]\right)\left(\tau_{1,2}\right)= \\
& \left(\theta\left[\begin{array}{l}
0 \\
0
\end{array}\right]^{4} \eta^{12}\right)\left(\tau_{1}\right) \Xi_{8}^{(2)}\left[0^{(2)}\right]\left(\tau_{2}\right) \equiv\left(\theta\left[\begin{array}{l}
0 \\
0
\end{array}\right]^{4} \eta^{12}\right)\left(\tau_{1}\right)\left(\theta\left[0^{(2)}\right]^{4} \Xi_{6}\left[0^{(2)}\right]\right)\left(\tau_{2}\right)
\end{aligned}
$$

is that the terms proportional to $f_{21}$ and to $F_{16}^{(1)}$ disappear. Here $\Xi_{6}\left[0^{(2)}\right]$ is the function found by D'Hoker and Phong in [11]. First, let us impose the condition to get rid of the terms proportional to $f_{21}$. This condition is satisfied if:

$$
a_{1} \frac{1}{3} F_{1}^{(2)}+a_{2} \frac{2}{3} F_{2}^{(2)}+a_{3} \frac{2}{3} F_{3}^{(2)}+a_{5}\left(\frac{4}{3} F_{88}^{(2)}-\frac{1}{3} F_{8}^{(2)}\right)=0 .
$$


This equation has a unique solution up to a scalar multiple:

$$
\left(a_{1}, a_{2}, a_{3}, a_{5}\right)=\lambda\left(\frac{16}{3}, \frac{16}{3},-4,1\right), \quad \lambda \in \mathbf{C} .
$$

To eliminate the terms proportional to $F_{16}^{(1)}$ the expression $\left(2 a_{4}+\frac{1}{2} a_{5}\right) F_{8}^{(2)}$ must vanish, so, from the solution 5.1, we obtain

$$
a_{4}=-\frac{1}{4} a_{5}=-\lambda \frac{1}{4}
$$

Thus the expression for the factorised measure is:

$$
\theta\left[\begin{array}{l}
0 \\
0
\end{array}\right]{ }^{4} \eta^{12} \lambda\left[\frac{16}{3} F_{1}^{(3)}+\frac{16}{3}\left(-F_{2}^{(3)}\right)+\left(F_{8}^{(3)}-4 F_{88}^{(3)}\right)\right]
$$

and it is of the form $\Xi_{8}^{(3)}\left[0^{(3)}\right]\left(\tau_{1,2}\right)=\left(\theta\left[\begin{array}{l}0 \\ 0\end{array}\right]^{4} \eta^{12}\right)\left(\tau_{1}\right)\left(\theta\left[0^{(2)}\right]^{4} \Xi_{6}\left[0^{(2)}\right]\right)$ if $\lambda=\frac{1}{16}$, as can be verified with a computer or using the classical theta formula. This solution for the form $\Xi_{8}^{(3)}\left[0^{(3)}\right]$ is, up to a term proportional to $J^{(3)}$, the same found in [7]. Using this basis, the theta constants in the function $\Xi_{8}^{(3)}\left[0^{(3)}\right]$ appear with higher power than using the one of [7]: the four functions $F_{1}^{(3)}, F_{3}^{(3)}, F_{8}^{(3)}$ and $F_{88}^{(3)}$ are polynomials in $\theta\left[\Delta^{(3)}\right]^{8}$ and they belong to $\operatorname{Sym}^{2}\left(\mathbf{1}+\mathbf{3 5}_{b}\right)$ and the $F_{2}^{(2)}$ is a polynomial in $\theta\left[\Delta^{(3)}\right]^{4}$ and belongs to $\operatorname{Sym}^{4}\left(\mathbf{1 5}_{a}\right)$. The final expression for the form $\Xi_{8}^{(3)}\left[0^{(3)}\right]$ is:

$$
\Xi_{8}^{(3)}\left[0^{(3)}\right]=\frac{1}{3} F_{1}^{(3)}+\frac{1}{3} F_{2}^{(3)}-\frac{1}{4} F_{3}^{(3)}-\frac{1}{64} F_{8}^{(3)}+\frac{1}{16} F_{88}^{(3)},
$$

and it is completely equivalent to the one determined in [7]:

$$
\Xi_{8}^{(3)}\left[0^{(3)}\right]=\frac{1}{3} F_{1}^{(3)}+\frac{1}{3} F_{2}^{(3)}-\frac{1}{4} F_{3}^{(3)}-G_{3}^{(3)}\left[0^{(3)}\right]
$$

In fact, they differ for a multiple of the form $J^{(3)}$ (that vanishes on the whole $\mathbf{H}_{3}$ ), precisely the new expression is equal the old one $-\frac{5}{448} J^{(3)}$, as it can be computed using the results of Table 4. This procedure will be explained in detail for the genus four case in the next Section. So we can use this two expressions to show that the form $G_{3}^{(3)}\left[0^{(3)}\right]$ is polynomial in $\theta\left[\Delta^{(3)}\right]^{8}$ :

$$
G_{3}^{(3)}\left[0^{(3)}\right]=\frac{1}{64} F_{8}^{(3)}-\frac{1}{16} F_{88}^{(3)}-\frac{5}{448}\left(8 F_{16}^{(3)}-F_{8}^{(3)}\right)
$$

We include also the form $J^{(3)}$ because it is zero as a function of $\tau \in \mathbf{H}_{3}$ and using a computer to perform the computations one has to add explicitly this fact. 


\section{Genus four case}

For the genus four case we repeat the method used for the genus three in the previous Section. In Section 3.2 we found seven linear independent $\mathrm{O}^{+}$-invariants that form a basis. We can now search a linear combination that also satisfies the factorisation constraints:

$$
\Xi_{8}^{(4)}\left[0^{(4)}\right]=a_{1} F_{1}^{(4)}+a_{2} F_{2}^{(4)}+a_{3} F_{3}^{(4)}+a_{4} F_{8}^{(4)}+a_{5} F_{88}^{(4)}+a_{6} F_{16}^{(4)}+a_{7} G_{3}^{(4)}\left[0^{(4)}\right]
$$

where $G_{3}^{(4)}\left[0^{(4)}\right]$ is the function defined in $\left[3.2\right.$, or in the notations of Grushevsky $P_{3,2}^{(4)}$. In this case we also use the $F_{16}^{(4)}$ because in $g=4$ it is independent from $F_{8}^{(4)}$, i.e. the expression $J^{(4)}$ is not identically zero on the whole $\mathbf{H}_{4}$, but just on the Jacobi locus.

6.1 The restriction of $\Xi_{8}^{(4)}\left[0^{(4)}\right]$ on $\mathbf{H}_{1} \times \mathbf{H}_{3}$

The restriction on $\mathbf{H}_{1} \times \mathbf{H}_{3}$ of the function $G_{3}^{(4)}\left[0^{(4)}\right]$ was found in [8]:

$$
\begin{aligned}
G_{3}^{(4)}\left[0^{(4)}\right]\left(\tau_{1,3}\right)= & \theta\left[\begin{array}{l}
0 \\
0
\end{array}\right]^{4}\left(\tau_{1}\right)\left[\frac{1}{3} f_{21}\left(\tau_{1}\right)\left(G_{2}^{(3)}\left[0^{(3)}\right]+8 G_{3}^{(3)}\left[0^{(3)}\right]\right)\left(\tau_{3}\right)\right. \\
& \left.-\eta^{12}\left(\tau_{1}\right)\left(G_{2}^{(3)}\left[0^{(3)}\right]+6 G_{3}^{(3)}\left[0^{(3)}\right]\right)\left(\tau_{3}\right)\right]
\end{aligned}
$$

this follows also from Theorem 15 of [17]. The modular forms $G_{3}^{(3)}\left[0^{(3)}\right]$ and $G_{2}^{(3)}\left[0^{(3)}\right]$ are defined in [7] and [8] respectively. Thus the factorisation of the expression (5) for a reducible period matrix of the form $\tau_{1,3}=\left(\begin{array}{cc}\tau_{1} & 0 \\ 0 & \tau_{3}\end{array}\right)$ is:

$$
\begin{aligned}
& \left(a_{1} F_{1}^{(4)}+a_{2} F_{2}^{(4)}+a_{3} F_{3}^{(4)}+a_{4} F_{8}^{(4)}+a_{5} F_{88}^{(4)}+a_{6} F_{16}^{(4)}+a_{7} G_{3}^{(4)}\left[0^{(4)}\right]\right)\left(\tau_{1,3}\right) \\
& =\theta\left[\begin{array}{l}
0 \\
0
\end{array}\right]^{4}\left[a_{1}\left(\frac{1}{3} f_{21}+\eta^{12}\right) F_{1}^{(3)}+a_{2}\left(\frac{2}{3} f_{21}-\eta^{12}\right) F_{2}^{(3)}+a_{3} \frac{2}{3} f_{21} F_{3}^{(3)}\right]+a_{4} 2 F_{16}^{(1)} F_{8}^{(3)} \\
& +a_{5}\left[\theta\left[\begin{array}{l}
0 \\
1
\end{array}\right]^{4} f_{21}\left(\frac{4}{3} F_{88}^{(3)}-\frac{1}{3} F_{8}^{(3)}\right)+\theta\left[\begin{array}{l}
0 \\
1
\end{array}\right]^{4} \eta^{12}\left(-4 F_{88}^{(3)}+F_{8}^{(3)}\right)+\frac{1}{2} F_{16}^{(1)} F_{8}^{(3)}\right] \\
& +a_{6} F_{16}^{(1)} F_{16}^{(3)}+a_{7}\left[\theta\left[\begin{array}{l}
0 \\
0
\end{array}\right]^{4} f_{21}\left(\frac{1}{3} G_{2}^{(3)}\left[0^{(3)}\right]+\frac{8}{3} G_{3}^{(3)}\left[0^{(3)}\right]\right)\right. \\
& \left.+\theta\left[\begin{array}{l}
0 \\
0
\end{array}\right]^{4} \eta^{12}\left(-G_{2}^{(3)}\left[0^{(3)}\right]-6 G_{3}^{(3)}\left[0^{(3)}\right]\right)\right] .
\end{aligned}
$$


The terms proportional to $f_{21}$ disappear if:

$$
\begin{aligned}
& a_{1} \frac{1}{3} F_{1}^{(3)}+a_{2} \frac{2}{3} F_{2}^{(3)}+a_{3} \frac{2}{3} F_{3}^{(3)}+a_{5}\left(\frac{4}{3} F_{88}^{(3)}-\frac{1}{3} F_{8}^{(3)}\right) \\
& +a_{7} \frac{1}{3}\left(G_{2}^{(3)}\left[0^{(3)}\right]+8 G_{3}^{(3)}\left[0^{(3)}\right]\right)=0 .
\end{aligned}
$$

This equation has a unique solution up to a scalar multiple:

$$
\left(a_{1}, a_{2}, a_{3}, a_{5}, a_{7}\right)=\lambda\left(-\frac{56}{5},-\frac{112}{5}, \frac{42}{5},-\frac{21}{5}, \frac{168}{5}\right), \quad \lambda \in \mathbf{C} .
$$

The term proportional to $F_{16}^{(1)}$ vanishes if:

$$
a_{4} 2 F_{8}^{(3)}+a_{5} \frac{1}{2} F_{8}^{(3)}+a_{6} F_{16}^{(3)}=0 .
$$

This equation has infinitely many solutions. Due to the vanishing of $J^{(3)}$ on the whole Siegel upper half space we can rewrite the previous equation as $\left(2 a_{4}+\frac{1}{2} a_{5}+\frac{1}{8} a_{6}\right) F_{8}=$ 0 , which has solution $a_{4}=-\frac{a_{5}}{4}-\frac{a_{6}}{16}$ with $a_{6} \in \mathrm{C}$. For any choice of $a_{6}$ an additive term proportional to $J^{(4)}$ appears in the expression of $\Xi_{8}^{(4)}\left[0^{(4)}\right]$ and precisely it is $\frac{a_{6}}{16}\left(16 F_{16}^{(4)}-F_{8}^{(4)}\right)$. In this sense the form $\Xi_{8}^{(4)}\left[0^{(4)}\right]$ is unique up to a term proportional to $J^{(4)}$, as proved in [10]. Thus, we can choose $a_{6}=0$ and $a_{4}=-\frac{1}{4} a_{5}$. The request for the restriction to be of the form $\Xi_{8}^{(4)}\left[0^{(4)}\right]\left(\tau_{1,3}\right)=\left(\theta\left[\begin{array}{l}0 \\ 0\end{array}\right]^{4} \eta^{12}\right)\left(\tau_{1}\right) \Xi_{8}^{(3)}\left[0^{(3)}\right]\left(\tau_{3}\right)$ fixes the value of $\lambda=-\frac{5}{336}$. This follow from the condition:

$$
\begin{aligned}
\theta\left[{ }_{0}^{0}\right]^{4} \eta^{12} \lambda[ & -\frac{56}{5} F_{1}^{(3)}-\frac{112}{5}\left(-F_{2}^{(3)}\right)+\frac{21}{5}\left(F_{8}^{(3)}-4 F_{88}^{(3)}\right) \\
& \left.+\frac{168}{5}\left[-\left(G_{2}^{(3)}\left[0^{(3)}\right]+6 G_{3}^{(3)}\left[0^{(3)}\right]\right)\right]\right]=\theta\left[{ }_{0}^{0}\right]^{4} \eta^{12} \Xi_{8}^{(3)}\left[0^{(3)}\right],
\end{aligned}
$$

and, using again the fact that $J^{(3)}$ identically vanishes, we obtain $\lambda=-\frac{5}{336}$.

The above discussion shows that the form $\Xi_{8}^{(4)}\left[0^{(4)}\right]$ is:

$$
\Xi_{8}^{(4)}\left[0^{(4)}\right]=\frac{1}{6} F_{1}^{(4)}+\frac{1}{3} F_{2}^{(4)}-\frac{1}{8} F_{3}^{(4)}+\frac{1}{64} F_{8}^{(4)}-\frac{1}{16} F_{88}^{(4)}-\frac{1}{2} G_{3}^{(4)}\left[0^{(4)}\right],
$$

which, for the uniqueness (up to a multiple of $J^{(4)}$ ) of the form $\Xi_{8}^{(4)}\left[0^{(4)}\right]$, is equivalent to the one found in [8]:

$$
\Xi_{8}^{(4)}\left[0^{(4)}\right]=\frac{1}{6} F_{1}^{(4)}+\frac{1}{3} F_{2}^{(4)}-\frac{1}{8} F_{3}^{(4)}-\frac{1}{2} G_{3}^{(4)}\left[0^{(4)}\right]+4 G_{4}^{(4)}\left[0^{(4)}\right] .
$$

This two expressions must be equated and they could differ just for a multiple of the Schottky relation $J^{(4)}$. Calling the form $\Xi_{8}^{(4)}\left[0^{(4)}\right]$ (6) found in this work $\Xi_{8}^{(4)}\left[0^{(4)}\right]_{D P}$ 
and the (7) of [8] $\Xi_{8}^{(4)}\left[0^{(4)}\right]_{C D G}$ we can write:

$$
\Xi_{8}^{(4)}\left[0^{(4)}\right]_{D P}=\Xi_{8}^{(4)}\left[0^{(4)}\right]_{C D G}+a J^{(4)} .
$$

Summing over the 136 even theta characteristics and using the results of Table 5 we find:

$$
\frac{45}{56} J^{(4)}=\frac{12}{7} J^{(4)}+136 a J^{(4)}
$$

from which it follows that $a=-3 / 448$. This shows that the modular form $G_{4}^{(4)}\left[0^{(4)}\right]$ is, actually, polynomial in $\theta\left[\Delta^{(4)}\right]^{8}$ :

$$
\begin{aligned}
G_{4}^{(4)}\left[0^{(4)}\right] & =\frac{1}{256} F_{8}^{(4)}-\frac{1}{64} F_{88}^{(4)}+\frac{3}{1792} J^{(4)} \\
& =\frac{1}{448} F_{8}^{(4)}-\frac{1}{64} F_{88}^{(4)}+\frac{3}{112} F_{16}^{(4)} .
\end{aligned}
$$

\subsubsection{Remark}

Recently, Oura has proved, as a consequence of the results in [26], that the space of modular forms with respect to the subgroup $\Gamma_{4}(1,2)$ of weight 8 , quadratic in the theta constants, has dimension no bigger than 7 , $\operatorname{dim} M_{8}^{\theta^{2}}\left(\Gamma_{4}(2)\right)^{O^{+}} \leq 7$. The computations in the previous Section show that this dimension is precisely seven:

$$
\operatorname{dim} M_{8}^{\theta^{2}}\left(\Gamma_{4}(2)\right)^{O^{+}}=7 .
$$

Moreover, in [27] it is proved that the space of cusp forms $\left[\Gamma_{4}(1,2), 8\right]_{0}$, in which $\Xi_{8}^{(4)}\left[0^{(4)}\right]$ lies, has dimension two. From this, it follows the uniqueness (up to a multiple of $J^{(4)}$ ) of the form $\Xi_{8}^{(4)}\left[0^{(4)}\right]$ (as explained in [27] and [18]) and not just in a weakened form, i.e. assuming polynomiality in the theta constants, as in [10].

\section{Genus five case}

In Section 3.3 we found eight linear independent $\mathrm{O}^{+}$-invariants that form a basis. Their general linear combination is:

$$
\begin{aligned}
\Xi_{8}^{(5)}\left[0^{(5)}\right] & =a_{1} F_{1}^{(5)}+a_{2} F_{2}^{(5)}+a_{3} F_{3}^{(5)}+a_{4} F_{8}^{(5)}+a_{5} F_{88}^{(5)} \\
& +a_{6} F_{16}^{(5)}+a_{7} G_{3}^{(5)}\left[0^{(5)}\right]+a_{8} G_{4}^{(5)}\left[0^{(5)}\right] .
\end{aligned}
$$

We will search for eight coefficients $a_{i}$ such that this expression satisfies the right factorisation. 
7.1 The restriction of $G_{3}^{(5)}\left[0^{(5)}\right]$ and $G_{4}^{(5)}\left[0^{(5)}\right]$ on $\mathbf{H}_{1} \times \mathbf{H}_{4}$

These restrictions follow quite directly from the Theorem of Grushevsky, identifying $G_{4}^{(5)}\left[0^{(5)}\right]$ with $P_{4,1}^{(5)}$ and $G_{3}^{(5)}\left[0^{(5)}\right]$ with $P_{3,2}^{(5)}$. For the function $G_{3}^{(5)}\left[0^{(5)}\right]$ we get:

$$
\begin{aligned}
P_{3,2}^{(5)}\left(\tau_{1,4}\right) & =P_{0,16}^{(1)}\left(\tau_{1}\right) P_{3,2}^{(4)}\left(\tau_{4}\right)+P_{1,8}^{(1)}\left(\tau_{1}\right) P_{2,4}^{(4)}\left(\tau_{4}\right)+7 P_{1,8}^{(1)}\left(\tau_{1}\right) P_{3,2}^{(4)}\left(\tau_{4}\right) \\
& =\theta\left[\begin{array}{l}
0 \\
0
\end{array}\right]^{4}\left(\frac{8}{3} f_{21}-6 \eta^{12}\right)\left(\tau_{1}\right) G_{3}^{(4)}\left[0^{(4)}\right]\left(\tau_{4}\right)+\theta\left[\begin{array}{l}
0 \\
0
\end{array}\right]^{4}\left(\frac{1}{3} f_{21}-\eta^{12}\right)\left(\tau_{1}\right) G_{2}^{(4)}\left[0^{(4)}\right]\left(\tau_{4}\right),
\end{aligned}
$$

where we used $P_{0,16}^{(1)}=\theta\left[0^{(1)}\right]^{4}\left(\frac{1}{3} f_{21}+\eta^{12}\right), P_{1,8}^{(1)}=\theta\left[0^{(1)}\right]^{4}\left(\frac{1}{3} f_{21}-\eta^{12}\right)$ and $P_{2,4}^{(4)}=$ $G_{2}^{(4)}\left[0^{(4)}\right]$. For $G_{4}^{(5)}\left[0^{(5)}\right]$ we get:

$$
\begin{aligned}
P_{4,1}^{(5)}\left(\tau_{1,4}\right) & =P_{0,16}^{(1)}\left(\tau_{1}\right) P_{4,1}^{(4)}\left(\tau_{4}\right)+P_{1,8}^{(1)}\left(\tau_{1}\right) P_{3,2}^{(4)}\left(\tau_{4}\right)+15 P_{1,8}^{(1)}\left(\tau_{1}\right) P_{4,1}^{(4)}\left(\tau_{4}\right) \\
& =\theta\left[\begin{array}{l}
0 \\
0
\end{array}\right]^{4}\left(\frac{16}{3} f_{21}-14 \eta^{12}\right)\left(\tau_{1}\right) G_{4}^{(4)}\left[0^{(4)}\right]\left(\tau_{4}\right)+\theta\left[\begin{array}{l}
0 \\
0
\end{array}\right]^{4}\left(\frac{1}{3} f_{21}-\eta^{12}\right)\left(\tau_{1}\right) G_{3}^{(4)}\left[0^{(4)}\right]\left(\tau_{4}\right),
\end{aligned}
$$

where, as before, $P_{0,16}^{(1)}=\theta\left[0^{(1)}\right]^{4}\left(\frac{1}{3} f_{21}+\eta^{12}\right)$ and $P_{1,8}^{(1)}=\theta\left[0^{(1)}\right]^{4}\left(\frac{1}{3} f_{21}-\eta^{12}\right)$. These restrictions could be also determined using the method of isotropic subspaces, as in [7, or by direct computation using a computer. In the next Section it will be useful to use also $G_{2}^{(4)}\left[0^{(4)}\right]=\left(2 F_{1}^{(4)}+16 F_{2}^{(4)}-3 F_{3}^{(4)}\right) / 6$ (instead, at genus three we have $\left.G_{2}^{(3)}\left[0^{(3)}\right]=\left(2 F_{1}^{(3)}+8 F_{2}^{(3)}-3 F_{3}^{(3)}\right) / 6\right)$. 
7.2 The restriction of $\Xi_{8}^{(5)}\left[0^{(5)}\right]$ on $\mathbf{H}_{1} \times \mathbf{H}_{4}$

Using the results of the previous Section and of Section 4 the factorisation of the expression (9) for a reducible period matrix of the form $\tau_{1,4}=\left(\begin{array}{cc}\tau_{1} & 0 \\ 0 & \tau_{4}\end{array}\right)$ is:

$$
\begin{aligned}
& \left(a_{1} F_{1}^{(5)}+a_{2} F_{2}^{(5)}+a_{3} F_{3}^{(5)}+a_{4} F_{8}^{(5)}+a_{5} F_{88}^{(5)}\right. \\
& \left.+a_{6} F_{16}^{(5)}+a_{7} G_{3}^{(5)}\left[0^{(5)}\right]+a_{8} G_{4}^{(5)}\left[0^{(5)}\right]\right)\left(\tau_{1,4}\right)= \\
& {\left[a_{1} \theta\left[\begin{array}{l}
0 \\
0
\end{array}\right]^{4}\left(\frac{1}{3} f_{21}+\eta^{12}\right) F_{1}^{(4)}+a_{2} \theta\left[\begin{array}{l}
0 \\
0
\end{array}\right]^{4}\left(\frac{2}{3} f_{21}-\eta^{12}\right) F_{2}^{(4)}+a_{3} \frac{2}{3} \theta\left[\begin{array}{l}
0 \\
0
\end{array}\right]^{4} f_{21} F_{3}^{(4)}\right]+} \\
& a_{4} 2 F_{16}^{(1)} F_{8}^{(4)}+a_{5}\left[\theta\left[\begin{array}{l}
0 \\
1
\end{array}\right]^{4} f_{21}\left(\frac{4}{3} F_{88}^{(4)}-\frac{1}{3} F_{8}^{(4)}\right)+\theta\left[\begin{array}{l}
0 \\
1
\end{array}\right]^{4} \eta^{12}\left(-4 F_{88}^{(4)}+F_{8}^{(4)}\right)\right. \\
& \left.+\frac{1}{2} F_{16}^{(1)} F_{8}^{(4)}\right]+a_{6} F_{16}^{(1)} F_{16}^{(4)}+ \\
& a_{7} \theta\left[\begin{array}{l}
0 \\
0
\end{array}\right]^{4}\left[\frac{1}{3} f_{21}\left(G_{2}^{(4)}\left[0^{(4)}\right]+8 G_{3}^{(4)}\left[0^{(4)}\right]\right)+\eta^{12}\left(-G_{2}^{(4)}\left[0^{(4)}\right]-6 G_{3}^{(4)}\left[0^{(4)}\right]\right)\right]+ \\
& a_{8} \theta\left[\begin{array}{l}
0 \\
0
\end{array}\right]^{4}\left[f_{21}\left(\frac{16}{3} G_{4}^{(4)}\left[0^{(4)}\right]+\frac{1}{3} G_{3}^{(4)}\left[0^{(4)}\right]\right)+\eta^{12}\left(-14 G_{4}^{(4)}\left[0^{(4)}\right]-G_{3}^{(4)}\left[0^{(4)}\right]\right)\right] .
\end{aligned}
$$

From the relations of paragraph 4.1 we obtain the condition for the vanishing of the terms proportional to $f_{21}$ :

$$
\begin{gathered}
a_{1} \frac{1}{3} F_{1}^{(4)}+a_{2} \frac{2}{3} F_{2}^{(4)}+a_{3} \frac{2}{3} F_{3}^{(4)}+a_{5}\left(\frac{4}{3} F_{88}^{(4)}-\frac{1}{3} F_{8}^{(4)}\right)+ \\
+a_{7} \frac{1}{3}\left(G_{2}^{(4)}\left[0^{(4)}\right]+8 G_{3}^{(4)}\left[0^{(4)}\right]\right)+a_{8} \frac{1}{3}\left(G_{3}^{(4)}\left[0^{(4)}\right]+16 G_{4}^{(4)}\left[0^{(4)}\right]\right)=0 .
\end{gathered}
$$

Again, this equation, using the fact that $J^{(4)}$ vanishes on the Jacobi locus (actually, one solves the equation modulo the Schottky relation $J^{(4)}$ ), has an unique solution, up to a scalar multiple:

$$
\left(a_{1}, a_{2}, a_{3}, a_{5}, a_{7}, a_{8}\right)=\lambda\left(-\frac{14}{3},-\frac{56}{3}, \frac{7}{2},-7,14,-112\right), \quad \lambda \in \mathbf{C} .
$$

The term proportional to $F_{16}^{(1)}$ vanishes if:

$$
a_{4} 2 F_{8}^{(4)}+a_{5} \frac{1}{2} F_{8}^{(4)}+a_{6} F_{16}^{(4)}=0 .
$$

As for $g=4$ this equation has infinitely many solutions and using again the fact that $J^{(4)}$ vanishes on the Jacobi locus we obtain $\left(2 a_{4}+\frac{1}{2} a_{5}+\frac{1}{16} a_{6}\right) F_{8}^{(4)}=0$, which has solution $a_{4}=-\frac{a_{5}}{4}-\frac{a_{6}}{32}$, with $a_{6} \in \mathbf{C}$. For any choice of the coefficient $a_{6}$ the additive term $\frac{a_{6}}{32}\left(32 F_{16}^{(5)}-F_{8}^{(5)}\right)$ appears in $\Xi_{8}^{(5)}\left[0^{(5)}\right]$. However, in genus five this 
term vanishes just on the locus of trigonal curves and not on the whole Jacobi locus. This shows how the uniqueness of the form $\Xi_{8}^{(5)}\left[0^{(5)}\right]$ can not be longer assured by the three constraints of Section 2 on $J_{5}$, as also pointed out in [27]. Thus, we can choose $a_{6}=0$ and $a_{4}=-\frac{1}{4} a_{5}$. The request for the restriction to be of the form $\Xi_{8}^{(5)}\left[0^{(5)}\right]\left(\tau_{1,4}\right)=\left(\theta\left[\begin{array}{l}0 \\ 0\end{array}\right]^{4} \eta^{12}\right)\left(\tau_{1}\right) \Xi_{8}^{(4)}\left[0^{(4)}\right]\left(\tau_{4}\right)$ means that:

$$
\begin{aligned}
&\left(\theta\left[\begin{array}{l}
0 \\
0
\end{array}\right]^{4} \eta^{12}\right)\left(\tau_{1}\right) \lambda[ a_{1} F_{1}^{(4)}-a_{2} F_{2}^{(4)}+a_{5}\left(F_{8}^{(4)}-4 F_{88}^{(4)}\right) \\
&\left.+a_{7}\left(-G_{2}^{(4)}\left[0^{(4)}\right]-6 G_{3}^{(4)}\left[0^{(4)}\right]\right)+a_{8}\left(-14 G_{4}^{(4)}\left[0^{(4)}\right]-G_{3}^{(4)}\left[0^{(4)}\right]\right)\right] \\
&=\left(\theta\left[\begin{array}{l}
0 \\
0
\end{array}\right]^{4} \eta^{12}\right)\left(\tau_{1}\right) \Xi_{8}^{(4)}\left[0^{(4)}\right],
\end{aligned}
$$

where $\Xi_{8}^{(4)}\left[0^{(4)}\right]$ is the function found in Section 6, and this should fix the constant $\lambda$. Therefore, we impose:

$$
\begin{aligned}
\theta\left[\begin{array}{l}
0 \\
0
\end{array}\right]^{4} \eta^{12} \lambda & {\left[-\frac{14}{3} F_{1}^{(4)}-\frac{56}{3}\left(-F_{2}^{(4)}\right)-7\left(F_{8}^{(4)}-4 F_{88}^{(4)}\right)+14\left(-G_{2}^{(4)}\left[0^{(4)}\right]-6 G_{3}^{(4)}\left[0^{(4)}\right]\right)\right.} \\
& \left.-112\left(-14 G_{4}^{(4)}-G_{3}^{(4)}\right)\right]=\theta\left[\begin{array}{l}
0 \\
0
\end{array}\right]^{4} \eta^{12}\left(\Xi_{8}^{(4)}\left[0^{(4)}\right]+\Lambda J^{(4)}\right)
\end{aligned}
$$

this equation has solution $\lambda=-\frac{1}{56}$ and $\Lambda=-\frac{3}{64}$. Actually, using $\lambda=-\frac{1}{56}$ and summing over all the even characteristics one finds that the expression in the square brackets on the left and the $\Xi_{8}^{(4)}\left[0^{(4)}\right]$ on the right sides of the previous equation differ by $-\frac{3}{64} J^{(4)}$. Thus, in genus five the function $\Xi_{8}^{(5)}\left[0^{(5)}\right]$ satisfying the three constraints on the Jacobi locus is:

$$
\Xi_{8}^{(5)}\left[0^{(5)}\right]=\frac{1}{12} F_{1}^{(5)}+\frac{1}{3} F_{2}^{(5)}-\frac{1}{16} F_{3}^{(5)}-\frac{1}{32} F_{8}^{(5)}+\frac{1}{8} F_{88}^{(5)}-\frac{1}{4} G_{3}^{(5)}\left[0^{(5)}\right]+2 G_{4}^{(5)}\left[0^{(5)}\right]
$$

Note that also for the solution found in [27] the correct restriction holds if one restrict to $J_{4}$.

\subsection{The constraint on $\mathbf{H}_{2} \times \mathbf{H}_{3}$}

Now we consider the restriction of the function $\Xi_{8}^{(5)}\left[0^{(5)}\right]$ to $\mathbf{H}_{2} \times \mathbf{H}_{3}$ and this, to satisfy the factorization constraint of Section 2, must be equal to the product $\Xi_{8}^{(2)}\left[0^{(2)}\right] \Xi_{8}^{(3)}\left[0^{(3)}\right]$ i.e. the genus two times the genus three measure.

In order to obtain the restriction of $\Xi_{8}^{(5)}\left[0^{(5)}\right]$ we need the restriction of the eight 
basis functions. We have:

$$
\begin{aligned}
F_{1 \mid \Delta_{2,3}}^{(5)} & =F_{1}^{(2)} F_{1}^{(3)} \\
F_{2 \mid \Delta_{2,3}}^{(5)} & =F_{2}^{(2)} F_{2}^{(3)} \\
F_{3 \mid \Delta_{2,3}}^{(5)} & =F_{3}^{(2)} F_{3}^{(3)} \\
F_{8 \mid \Delta_{2,3}}^{(5)} & =F_{8}^{(2)} F_{8}^{(3)} \\
F_{16 \mid \Delta_{2,3}}^{(5)} & =F_{16}^{(2)} F_{16}^{(3)} \\
F_{88 \mid \Delta_{2,3}}^{(5)} & =F_{1}^{(2)}\left(\frac{16}{3} F_{88}^{(3)}-\frac{4}{3} F_{8}^{(3)}\right)+F_{2}^{(2)}\left(\frac{32}{3} F_{88}^{(3)}-\frac{8}{3} F_{8}^{(3)}\right) \\
& +F_{3}^{(2)}\left(-8 F_{88}^{(3)}+2 F_{8}^{(3)}\right)+F_{16}^{(2)} F_{8}^{(3)} \\
G_{3 \mid \Delta_{2,3}}^{(5)} & =G_{0}^{(2)} G_{3}^{(3)}+7 G_{1}^{(2)} G_{3}^{(3)}+G_{1}^{(2)} G_{2}^{(3)}+42 G_{2}^{(2)} G_{3}^{(3)}+9 G_{2}^{(2)} G_{2}^{(3)}+G_{2}^{(2)} G_{1}^{(3)} \\
& =F_{1}^{(2)}\left(\frac{1}{3} F_{1}^{(3)}+\frac{8}{3} F_{2}^{(3)}-\frac{2}{3} F_{3}^{(3)}+\frac{1}{8} F_{8}^{(3)}-\frac{1}{2} F_{88}^{(3)}\right) \\
& +F_{2}^{(2)}\left(\frac{4}{3} F_{1}^{(3)}+8 F_{2}^{(3)}-\frac{7}{3} F_{3}^{(3)}+\frac{7}{16} F_{8}^{(3)}-\frac{7}{4} F_{88}^{(3)}\right) \\
& +F_{3}^{(2)}\left(-\frac{2}{3} F_{1}^{(3)}-\frac{14}{3} F_{2}^{(3)}+\frac{5}{4} F_{3}^{(3)}-\frac{7}{32} F_{8}^{(3)}+\frac{7}{8} F_{88}^{(3)}\right), \\
& =G_{1}^{(2)} G_{3}^{(3)}+21 G_{2}^{(2)} G_{3}^{(3)}+G_{2}^{(2)} G_{2}^{(3)} \\
& =F_{1}^{(2)}\left(\frac{1}{9} F_{1}^{(3)}+\frac{4}{9} F_{2}^{(3)}-\frac{1}{6} F_{3}^{(3)}+\frac{3}{32} F_{8}^{(3)}-\frac{3}{8} F_{88}^{(3)}\right) \\
& +F_{2}^{(2)}\left(\frac{2}{9} F_{1}^{(3)}+\frac{8}{9} F_{2}^{(3)}-\frac{1}{3} F_{3}^{(3)}+\frac{7}{32} F_{8}^{(3)}-\frac{7}{8} F_{88}^{(3)}\right) \\
& +F_{3}^{(2)}\left(-\frac{1}{6} F_{1}^{(3)}-\frac{2}{3} F_{2}^{(3)}+\frac{1}{4} F_{3}^{(3)}-\frac{19}{128} F_{8}^{(3)}+\frac{19}{32} F_{88}^{(3)}\right) . \\
G_{4 \mid \Delta_{2,3}}(3) & \\
F^{(3)} & \\
&
\end{aligned}
$$

The first five relations follow quite easly from the definitions and the classical theta formula. The sixth is longer to prove in the same manner and it can be obtained using software like Mathematica. The last two follow from Theorem 15 of [17]. In [9], using the relations between the lattice theta series and the classical theta constants, another proof of the first six restrictions and of the one of $G_{4}^{(5)}\left[0^{(5)}\right]$ will be given.

We can now obtain the restriction of the form $\Xi_{8}^{(5)}\left[0^{(5)}\right]$ :

$$
\begin{aligned}
\Xi_{8}^{(5)}\left[0^{(5)}\right]\left(\tau_{2,3}\right)= & \left(\frac{2}{3} F_{1}^{(2)}+\frac{1}{3} F_{2}^{(2)}-\frac{1}{2} F_{3}^{(2)}\right) \\
& \cdot\left(\frac{1}{3} F_{1}^{(3)}+\frac{1}{3} F_{2}^{(3)}-\frac{1}{4} F_{3}^{(3)}-\frac{1}{64} F_{8}^{(3)}+\frac{1}{16} F_{88}^{(3)}\right) \\
= & \Xi_{8}^{(2)}\left[0^{(2)}\right]\left(\tau_{2}\right) \Xi_{8}^{(3)}\left[0^{(3)}\right]\left(\tau_{3}\right) .
\end{aligned}
$$

Therefore the modular form $\Xi_{8}^{(5)}\left[0^{(5)}\right]$ on $\Gamma_{5}(1,2)$ of weight 8 , defined in 7.2 , satisfies 
all the factorization constraints in genus five.

\subsubsection{Remark}

It is interesting to investigate the possibility to apply a similar procedure to the genus six case. However, in this case we have no indication about the dimension of the space of $\mathrm{O}^{+}$-invariant modular forms, not even if one restricts oneself to the polynomial in the theta constants part of the ring of modular forms. Tentatively one can try to built some invariants employing the action of the generators of the symplectic group, as we did in Section 3.1 to define the function $F_{88}^{(3)}$, and search a linear combination among them with the correct restriction. These topics will be considered for a future work.

\subsection{On the dimensions of certain space of modular forms}

In Sections 6 and 7 we considered the space of the modular forms of weight 8 with respect the group $\Gamma_{g}(1,2)$. In particular we focused on the modular forms polynomial in theta constants. In order to find the forms $\Xi_{8}^{(g)}\left[0^{(g)}\right]$ that factorise in the right way we searched for a basis for these spaces and this allowed us to find the dimensions of the spaces. We summarise these results in the following (cf. Remark 3.3.3):

Proposition 1 For the space $M_{8}^{\theta^{2}}\left(\Gamma_{4}(2)\right)^{O^{+}}, M_{8}^{\theta^{2}}\left(\Gamma_{5}(2)\right)^{O^{+}}$and $M_{8}^{\theta}\left(\Gamma_{5}(2)\right)^{O^{+}}$the following equalities hold:

$$
\begin{aligned}
& \operatorname{dim} M_{8}^{\theta^{2}}\left(\Gamma_{4}(2)\right)^{O^{+}}=7, \\
& \operatorname{dim} M_{8}^{\theta^{2}}\left(\Gamma_{5}(2)\right)^{O^{+}}=7, \\
& \operatorname{dim} M_{8}^{\theta}\left(\Gamma_{5}(2)\right)^{O^{+}}=8 .
\end{aligned}
$$

\section{The vanishing of the cosmological constant}

In this Section we reinterpret the vanishing of the cosmological constant on the light of the group representation theory. In Section 2 we pointed out that the $\mathrm{O}^{+}$invariants belong to the 1 and $\sigma_{\theta}$ representations. For the case $g \leq 5$ we know that the only $\operatorname{Sp}(2 g)$ invariants are $F_{16}^{(g)}$ and $F_{8}^{(g)}$ (they are not independent for $g=3$ ) and they form a basis for the $\mathbf{1}$ part of the space of the $O^{+}$-invarints. Let $\left\{e_{\sigma_{i}}\right\}_{i=1, \cdots, n_{\sigma_{\theta}}}$ be the basis for the $\sigma_{\theta}$ part. Then, an $O^{+}$-invariant decomposes in two 
parts: the first one lying in the representation 1 and the second one in the $\sigma_{\theta}$. Thus, if $f\left[0^{(g)}\right] \in M_{8}\left(\Gamma_{g}(2)\right)^{O^{+}}$, we can write $f\left[0^{(g)}\right]=a F_{8}^{(g)}+b F_{16}^{(g)}+\sum_{i}^{n_{\sigma_{\theta}}} c_{i} e_{\sigma_{i}}$, for $g \leq 5$. Acting on these functions with all the generators of the group $\operatorname{Sp}(2 g)$ and summing up the result at each step we obtain a $\mathrm{Sp}(2 g)$-invariant. We know that the unique $\mathrm{Sp}(2 g)$-invariants are the two functions $F_{8}^{(g)}$ and $F_{16}^{(g)}$ so that the $\sigma_{\theta}$ representation part gives no contribution to the sum. Therefore, if the function $f\left[0^{(g)}\right]$ contains a non trivial part proportional to $F_{8}^{(g)}$ or $F_{16}^{(g)}$, the result of the sum will be non zero.

The cosmological constant is the sum of the functions $\Xi_{8}^{(g)}\left[\Delta^{(g)}\right]$ over all the even characteristics. This sum is a $\operatorname{Sp}(2 g)$-invariant and it must then be proportional to a combination of $F_{8}^{(g)}$ and $F_{16}^{(g)}$. Thus the cosmological constant vanishes if this sum is zero. We now verify this for the genus three, four and five cases.

\subsection{Genus three}

In Table 4 we report the sums of each term appearing in the form $\Xi_{8}^{(3)}\left[0^{(3)}\right]$. These show that for the expression of the measure in the three bases (the one of 7] (CDG), the one in this work (DP) and the basis of [17] (Gr)) for the space of $O^{+}$-invariants we always obtain the vanishing of the cosmological constant (as expected) due to the vanishing of the form $J^{(3)}$.

\begin{tabular}{ccccc}
\hline Function & Sum & CDG & DP & Gr \\
\hline$F_{1}^{(3)}$ & $F_{16}^{(3)}$ & $\frac{1}{3}$ & $\frac{1}{3}$ & $\frac{1}{8}$ \\
$F_{2}^{(3)}$ & $8 F_{16}^{(3)}$ & $\frac{1}{3}$ & $\frac{1}{3}$ & 0 \\
$F_{3}^{(3)}$ & $F_{8}^{(3)}$ & $-\frac{1}{4}$ & $-\frac{1}{4}$ & 0 \\
$F_{8}^{(3)}$ & $36 F_{8}^{(3)}$ & 0 & $-\frac{1}{64}$ & 0 \\
$F_{88}^{(3)}$ & $8 F_{8}^{(3)}-8 F_{16}^{(3)}$ & 0 & $\frac{1}{16}$ & 0 \\
$G_{1}^{(3)}\left[0^{(3)}\right]$ & $F_{8}^{(3)}-F_{16}^{(3)}$ & 0 & 0 & $-\frac{1}{8}$ \\
$G_{2}^{(3)}\left[0^{(3)}\right]$ & $11 F_{16}^{(3)}-\frac{1}{2} F_{8}^{(3)}$ & 0 & 0 & $\frac{1}{4}$ \\
$G_{3}^{(3)}\left[0^{(3)}\right]$ & $\frac{1}{28}\left(13 F_{8}^{(3)}-76 F_{16}^{(3)}\right)$ & -1 & 0 & -1 \\
\hline Total & & $\frac{5}{7}\left(8 F_{16}^{(3)}-F_{8}^{(3)}\right)$ & $\frac{5}{16}\left(8 F_{16}^{(3)}-F_{8}^{(3)}\right)$ & $\frac{5}{7}\left(8 F_{16}^{(3)}-F_{8}^{(3)}\right)$ \\
\hline
\end{tabular}

Table 4

Sums of the terms appearing in $\Xi_{8}^{(3)}\left[0^{(3)}\right]$. In the third, fourth and fifth columns we report the coefficients of the $O^{+}$-invariants appearing in the expression of $\Xi_{8}^{(3)}\left[0^{(3)}\right]$ in the three basis. 


\subsection{Genus four}

As for the genus three case, we report in Table 5 the sums of each term appearing in the form $\Xi_{8}^{(4)}\left[0^{(4)}\right]$. Again, for the three equivalent bases of the space of $O^{+}$-invariants, the cosmological constant vanishes on the Jacobi locus due to the vanishing of the form $J^{(4)}$. It should be noted that the cosmological constant vanishes just on the moduli space of curves even if the forms $\Xi_{8}^{(4)}\left[\Delta^{(4)}\right]$ are well defined on the whole $\mathbf{H}_{4}$.

\begin{tabular}{ccccc}
\hline Function & Sum & CDG & DP & Gr \\
\hline$F_{1}^{(4)}$ & $F_{16}^{(4)}$ & $\frac{1}{6}$ & $\frac{1}{6}$ & $\frac{1}{16}$ \\
$F_{2}^{(4)}$ & $16 F_{16}^{(4)}$ & $\frac{1}{3}$ & $\frac{1}{3}$ & 0 \\
$F_{3}^{(4)}$ & $F_{8}^{(4)}$ & $-\frac{1}{8}$ & $-\frac{1}{8}$ & 0 \\
$F_{8}^{(4)}$ & $136 F_{8}^{(4)}$ & 0 & $\frac{1}{64}$ & 0 \\
$F_{88}^{(4)}$ & $32 F_{8}^{(4)}-32 F_{16}^{(4)}$ & 0 & $-\frac{1}{16}$ & 0 \\
$F_{16}^{(4)}$ & $136 F_{16}^{(4)}$ & 0 & 0 & 0 \\
$G_{1}^{(4)}\left[0^{(4)}\right]$ & $F_{8}^{(4)}-F_{16}^{(4)}$ & 0 & 0 & $-\frac{1}{16}$ \\
$G_{2}^{(4)}\left[0^{(4)}\right]$ & $43 F_{16}^{(4)}-\frac{1}{2} F_{8}^{(4)}$ & 0 & 0 & $\frac{1}{8}$ \\
$G_{3}^{(4)}\left[0^{(4)}\right]$ & $\frac{15}{7}\left(\frac{3}{4} F_{8}^{(4)}-5 F_{16}^{(4)}\right)$ & $-\frac{1}{2}$ & $-\frac{1}{2}$ & $-\frac{1}{2}$ \\
$G_{4}^{(4)}\left[0^{(4)}\right]$ & $\frac{29}{7} F_{16}^{(4)}-\frac{11}{56} F_{8}^{(4)}$ & 4 & 0 & 4 \\
\hline Total & & $\frac{12}{7}\left(16 F_{16}^{(4)}-F_{8}^{(4)}\right)$ & $\frac{45}{56}\left(16 F_{16}^{(4)}-F_{8}^{(4)}\right)$ & $\frac{12}{7}\left(16 F_{16}^{(4)}-F_{8}^{(4)}\right)$ \\
\hline
\end{tabular}

Table 5

Sums of the terms appearing in $\Xi_{8}^{(4)}\left[0^{(4)}\right]$. In the third, fourth and fifth columns we report the coefficients of the $O^{+}$-invariants appearing in the expression of $\Xi_{8}^{(4)}\left[0^{(4)}\right]$ in the three basis.

\subsection{Genus five}

As for the two previous cases we report in Table 6 the sums of each term appearing in the form $\Xi_{8}^{(5)}\left[0^{(5)}\right]$. In the Table the functions $G_{i}^{(5)}\left[0^{(5)}\right], i=0, \cdots, 5$, with $G_{0}^{(5)}\left[0^{(5)}\right] \equiv$ $F_{1}^{(5)}$, are the same as in [17]. In the genus five case the cosmological constant no longer vanishes neither on $J_{5}$. Actually, it was shown in [18] that the zero locus of $J^{(5)}$ is the locus of trigonal curves. Following [27], if we subtract from the forms $\Xi_{8}^{(5)}\left[0^{(5)}\right]$ the value of the cosmological constants divided by 528 , the number of the even characteristics in genus five, we obtain again a function satisfying the three constraints and, moreover, having zero cosmological constant. The correct 
factorization is due to the fact that the form $J^{(5)}$ vanishes when restrict both on $\mathbf{H}_{1} \times \mathbf{H}_{4}$ and on $\mathbf{H}_{2} \times \mathbf{H}_{3}$. Moreover, this consideration shows that in genus five the three constraints no longer assure the uniqueness of the form $\Xi_{8}^{(5)}\left[0^{(5)}\right]$ because we can always add a multiple of the Schottky relation that is not zero on $J_{5}$ obtaining another forms with the correct behaviour.

\begin{tabular}{cccc}
\hline Function & Sum & DP & Gr \\
\hline$F_{1}^{(5)}$ & $F_{16}^{(5)}$ & $\frac{1}{12}$ & $\frac{1}{32}$ \\
$F_{2}^{(5)}$ & $32 F_{16}^{(5)}$ & $\frac{1}{3}$ & 0 \\
$F_{3}^{(5)}$ & $F_{8}^{(5)}$ & $-\frac{1}{16}$ & 0 \\
$F_{8}^{(5)}$ & $528 F_{8}^{(5)}$ & $-\frac{1}{32}$ & 0 \\
$F_{88}^{(5)}$ & $128 F_{8}^{(5)}-128 F_{16}^{(5)}$ & $\frac{1}{8}$ & 0 \\
$F_{16}^{(5)}$ & $528 F_{16}^{(5)}$ & 0 & 0 \\
$G_{1}^{(5)}\left[0^{(5)}\right]$ & $F_{8}^{(5)}-F_{16}^{(5)}$ & 0 & $-\frac{1}{32}$ \\
$G_{2}^{(5)}\left[0^{(5)}\right]$ & $171 F_{16}^{(5)}-\frac{1}{2} F_{8}^{(5)}$ & 0 & $\frac{1}{16}$ \\
$G_{3}^{(5)}\left[0^{(5)}\right]$ & $\frac{173}{28} F_{8}^{(5)}-\frac{299}{7} F_{16}^{(5)}$ & $-\frac{1}{4}$ & $-\frac{1}{4}$ \\
$G_{4}^{(5)}\left[0^{(5)}\right]$ & $\frac{389}{7} F_{16}^{(5)}-\frac{43}{56} F_{8}^{(5)}$ & 2 & 2 \\
$G_{5}^{(5)}\left[0^{(5)}\right]$ & $-\frac{733}{217} F_{16}^{(5)}+\frac{475}{3472} F_{8}^{(5)}$ & 0 & -32 \\
\hline Total & & $\frac{51}{14}\left(32 F_{16}^{(5)}-F_{8}^{(5)}\right)$ & $\frac{1632}{217}\left(32 F_{16}^{(5)}-F_{8}^{(5)}\right)$ \\
\hline
\end{tabular}

Table 6

Sums of the terms appearing in $\Xi_{8}^{(5)}\left[0^{(5)}\right]$. In the third, fourth and fifth columns we report the coefficients of the $O^{+}$-invariants appearing in the expression of $\Xi_{8}^{(5)}\left[0^{(5)}\right]$ in the two basis.

\subsubsection{Remark}

The sums reported in Table 4, 5, and 6] can be computed using a computer and a software (for example, Mathematica), and in any case follow directly from Lemma 9 in 33 . 


\section{Conclusion}

In this work, enlightened by the representation theory of finite groups, we have obtained some new expressions of the superstring measure at genus three and four in which the classical theta functions appear at higher power than in previous works. For the genus five case we define a function that on the Jacobi locus has the correct behaviour with respect to the three constraints of Section 2. Although this form satisfies all the constraints it is far to be unique. In $g=5$ the constraints are not strong enough to characterize it completely. Moreover, the non normality of the ring of the modular forms in genus five and the possibility of some (not known) relations between the theta constants make it hard to prove the uniqueness. At this point some more insight in the physics leading to the formulations of the ansätze in Section 2 is necessary. Some checks for the proposed ansätze for the measure are considered by Morozov in [23|24]: he investigated the vanishing of 1,2,3-point functions. In [19] Grushevsky and Salvati Manni proved the vanishing of the 2-point function for $g=3$. Moreover, in [21] Matone and Volpato show how the chiral superstring amplitudes can be obtained through factorisation of the higher genus chiral measure. In [22] they also discuss the vanishing of the three-point amplitude at three loop. These facts open some general questions about the formulation of string theory in the perturbative approach. The ansätze of D'Hoker and Phong, modified as in [7], don't suffice to characterise the measure uniquely. Moreover, is the general form of the amplitudes (1), proposed by D'Hoker and Phong, from which one works out the expression of the measures correct? Some questions arise about this last point and about the fibration that led to (1), see [5] for a discussion. Again, the expression

for the forms $\Xi_{8}^{(g)}\left[0^{(g)}\right]$ found in [27] using the formalism of theta series open new questions about the uniqueness of the superstring measure.

These points seem to reopen some old problems about the general formulation of the perturbative approach to superstring theory.

\section{Acknowledgements}

I am grateful to Sergio L. Cacciatori and Bert van Geemen for several interesting and stimulating discussions, for some advices on how the superstring measure could be rewritten and for detailed comments on the first versions of this paper. I like to thank Vittorio Gorini for suggestions. I am also indebted with Riccardo Salvati Manni for explaining me some aspects about the dimensions of certain spaces of modular forms, for his comments on the draft and for a pleasant meeting in Milano. 


\section{A: Moduli space and Schottky problem}

Here we briefly summarise some topics about the moduli space of Riemann surfaces, for details see [37.

Let $C$ be a Riemann surface of genus $g$ and consider the homology group $H_{1}(C, \mathbf{Z}) \cong$ $\mathbf{Z}^{2 g}$. A symplectic basis of $H_{1}(C, \mathbf{Z})$ is a basis $\left\{\alpha_{1}, \cdots, \alpha_{g}, \beta_{1}, \cdots, \beta_{g}\right\}$ satisfying $\left(\alpha_{i}, \alpha_{j}\right)=\left(\beta_{i}, \beta_{j}\right)=0$ and $\left(\alpha_{i}, \beta_{j}\right)=\delta_{i j}$. Let $H^{0}\left(C, \Omega_{C}\right)$ be the $g$-dimensional complex vector space of holomorphic one forms on $C$. Given a path $\gamma \in C$ and an $\omega \in H^{0}\left(C, \Omega_{C}\right)$ one can compute the integral $\int_{\gamma} \omega$ and, if $\gamma$ is a closed path, the integral depends only on the homology class of $\gamma$. It can be shown that given a symplectic basis for $H_{1}(C, \mathbf{Z})$ then there is a unique basis $\left\{\omega_{1}, \cdots, \omega_{g}\right\}$ of $H^{0}\left(C, \Omega_{C}\right)$ such that $\int_{\alpha_{i}} \omega_{j}=\delta_{i j}$. We now use the $\beta_{j}$ to define a complex $g \times g$ matrix, the period matrix of $C, \tau=\left(\tau_{i j}\right) \in M_{g}(\mathbf{C})$ with $\tau_{i j}:=\int_{\beta_{i}} \omega_{j}$, where $\omega_{i}$ is an element of the basis of $H^{0}\left(C, \Omega_{C}\right)$ determined from the symplectic basis. Torelli's theorem asserts that one can recover the Riemann surface from its period matrix. The Schottky problem basically asks for equations which determine the period matrices of Riemann surfaces among all $g \times g$ matrices. Period matrices have two properties: they are symmetric and $\operatorname{Im}(\tau)$, the imaginary part of $\tau$, which is a symmetric, real, $g \times g$ matrix, defines a positive definite quadratic form on $\mathbf{R}^{g}:{ }^{t} x(\operatorname{Im} \tau) x>0$, for all $x \in \mathbf{R}^{g}$; briefly one writes $\operatorname{Im}(\tau)>0$. This leads to the definition of the Siegel upper half plane $\mathbf{H}_{g}:=\left\{\tau \in M_{g}(\mathbf{C}):{ }^{t} \tau=\tau, \operatorname{Im}(\tau)>0\right\}$. Thus if $\tau$ is the period matrix of a Riemann surface, then $\tau \in \mathbf{H}_{g}$. One can show that $\mathbf{H}_{g}$ is a complex manifold of dimension $\frac{1}{2} g(g+1)$.

To define the period matrix of a Riemann surface we had to choose a symplectic basis and two such basis are related by an element of the symplectic group $\Gamma_{g}$. The symplectic group acts on $\mathbf{H}_{g}$ and the period matrix of Riemann surfaces are a $\Gamma_{g^{-}}$ orbit in $\mathbf{H}_{g}$. Thus one can study the images of period matrices under the quotient map $\pi: \mathbf{H}_{g} \rightarrow A_{g}:=\Gamma_{g} \backslash \mathbf{H}_{g}$. The moduli space $M_{g}$ of Riemann surfaces is a variety whose points correspond to isomorphism classes of Riemann surfaces. Then we have a well defined holomorphic map: $j: M_{g} \rightarrow A_{g}$, $[X] \mapsto \Gamma_{g} \tau$, where $\tau$ is a period matrix of $X$. This map is injective from Torelli's theorem. The Schottky problem can now be reformulated as the problem of finding equations for the image of $j$.

Let $J_{g}^{0} \subset \mathbf{H}_{g}$ be the set of period matrices of Riemann surfaces. Its image in $A_{g}$ is 
$j\left(M_{g}\right)=\operatorname{Image}\left(J_{g}^{0} \rightarrow A_{g}=\Gamma_{g} \backslash \mathbf{H}_{g}\right)$. We have the diagram:

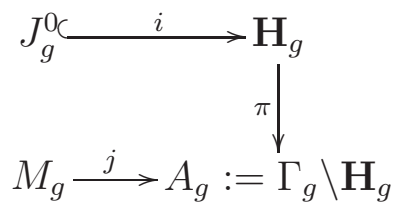

where $i$ is the immersion map of $J_{g}^{0}$ in $\mathbf{H}_{g}$ and $J\left(M_{g}\right)=\pi\left(i\left(J_{g}^{0}\right)\right)$. The subvariety $J_{g}^{0}$ and $j\left(M_{g}\right)$ are not closed and one defines the Jacobi locus $J_{g}$ as the closure of $J_{g}^{0}$ in $\mathbf{H}_{g}$. A $\tau \in \mathbf{H}_{g}$ will be called decomposable if $\tau$ lies in the $\Gamma_{g^{-}}$-orbit of matrices in diagonal block form. The set $J_{g}-J_{g}^{0}$ in $\mathbf{H}_{g}$ consists of decomposable matrices, the diagonal blocks being period matrices of Riemann surfaces of lower genus. From Teichmüller theory one knows that the subset $J_{g}$ is actually an irreducible subvariety of $\mathbf{H}_{g}$ of dimension $3 g-3$, for $g>1$ and for $g=1$ one has $\mathbf{H}_{1}=J_{1}=J_{1}^{0}$. The Table 1 shows that the Schottky problem is trivial for $g \leq 3$. This shows why for $g \leq 3$, as expected, the forms $\Xi_{8}^{(g)}\left[0^{(g)}\right]$ are defined on the whole $\mathbf{H}_{g}$.

\section{References}

[1] A. N. Andrianov and V. G. Zhuravlev, Modular Forms and Hecke Operators, American Mathematical Society, 1995.

[2] A. A. Belavin, V. G. Knizhnik, Algebraic Geometry and the Geometry of Quantum Strings, Phys. Lett. B 168 (1986) 201-206.

[3] A. A. Belavin, V. G. Knizhnik, Complex geometry and the theory of quantum string, Sov. Phys. JETP 64 (1986) 214-228; Zh. Eksp. Teor. Fiz. 91 (1986) 364-390.

[4] A. A. Belavin, V. G. Knizhnik, A. Morozov, A. Perelemov, Two- and three-loop amplitudes in bosonic string theory, Phys. Lett. 177 B (1986); ZhETF 43 (1986) 319.

[5] S. L. Cacciatori, F. Dalla Piazza, Two loop superstring amplitudes and $S_{6}$ representation, Lett. Math. Phys. 83 (2008) 127-138.

[6] S. L. Cacciatori, F. Dalla Piazza, Modular forms and superstrings amplitudes, to appear in Superstring Theory in the 21st Century. Horizons in World Physics. Volume 270. Editor Gerold B. Charney, Nova Publishers.

[7] S. L. Cacciatori, F. Dalla Piazza and B. van Geemen, Modular Forms and Three Loop Superstring Amplitudes, Nucl. Phys. B 800 (2008) 565-590.

[8] S. L. Cacciatori, F. Dalla Piazza and B. van Geemen, Genus four superstring measures, Lett. Math. Phys. 85 (2008) 185-193. 
[9] F. Dalla Piazza, D. Girola, and S. L. Cacciatori, Classical theta constants vs. lattice theta series, and super string partition functions, in preparation.

[10] F. Dalla Piazza and B. van Geemen, Siegel modular forms and finite symplectic groups, Adv. Theor. Math. Phys. 13 (2009), no. 6 (in press); arXiv:0804.0457.

[11] E. D'Hoker, D.H. Phong, Two-loop superstrings IV: The cosmological constant and modular forms, Nucl. Phys. B 639 (2002) 129-181.

[12] E. D'Hoker, D.H. Phong, Asyzygies, modular forms, and the superstring measure I, Nucl. Phys. B 710 (2005) 58-82.

[13] E. D'Hoker, D.H. Phong, Asyzygies, modular forms, and the superstring measure II, Nucl. Phys. B 710 (2005) 83-116.

[14] P. Dunin-Barkowski, A. Morozov, A. Sleptsov, Lattice Theta Constants versus Riemann Theta Constants and NSR Superstring Measures, JHEP10 (2009) 072.

[15] J.S. Frame, The classes and representations of the group of 27 lines and 28 bitangents, Annali di Mathematica Pura ed Applicata, 32 (1951) 83-119.

[16] J.S. Frame, Some characters of orthogonal groups over the field of two elements, In: Proc. of the Second Inter. Conf. on the Theory of Groups, Lecture Notes in Math., Vol. 372, pp. 298-314, Springer, 1974.

[17] S. Grushevsky, Superstring scattering amplitudes in higher genus, Commun. Math. Phys. 287 (2009) 749-767.

[18] S. Grushevsky and R. Salvati Manni, The superstring cosmological constant and the Schottky form in genus 5, to appear in Am. Jour. Math., e-Print: arXiv:0809.1391.

[19] S. Grushevsky and R. Salvati Manni, The vanishing of two-point functions for threeloop superstring scattering amplitudes, Commun. Math. Phys. 294 (2010), no. 2, 343352.

[20] N. Jacobson, Basic Algebra I. W.H. Freeman and Company, 1974.

[21] M. Matone, R. Volpato Getting superstring amplitudes by degenerating Riemann surfaces, e-Print: arXiv:1003.3452 [hep-th].

[22] M. Matone, R. Volpato Superstring measure and non-renormalization of the threepoint amplitude, Nucl. Phys. B 806 (2009) 735-747.

[23] A. Morozov, NSR measures on hyperelliptic locus and non-renormalization of 1,2,3point functions, Phys. Lett. B 664 (2008) 116-122.

[24] A. Morozov, NSR Superstring Measures Revisited, JHEP 0805 (2008) 086.

[25] A. Morozov, Explicit formulae for one, two, three and four loop string amplitudes, Phys. Lett. B 184 (1987) 171-186. 
[26] G. Nebe, Kneser-Hecke-operators in coding theory, Abh. Math. Sem. Univ. Hamburg 76 (2006), 79-90.

[27] M. Oura, C. Poor, R. Salvati Manni, and D. S. Yuen, Modular form of weight 8 for $\Gamma_{g}(1,2)$, Math. Ann. 346 (2010) no.2, 477-498.

[28] C. Poor, Schottky's form and the hyperelliptic locus, Proc. Am. Math. Soc. 124 (1996), 1987-1991.

[29] H. E. Rauch and A. Lebowitz, Elliptic Functions, Theta Funcitons, and Riemann Surfaces. The Williams \& Wilkins Company, 1974.

[30] B. Runge, On Siegel modular forms, part I, J. Reine Angew. Math. 436 (1993) 57-85.

[31] B. Runge, On Siegel modular forms, part II, Nagoya Math. J. 138 (1995) 179-197.

[32] B. E. Sagan, The Symmetric Group. Representations, Combinatorial Algorithms, and Symmetric Funtions. Springer 2001.

[33] R. Salvati Manni, Remarks on superstring amplitudes in higher genus, Nucl. Phys. B801 (2008) 163-173.

[34] R. Salvati Manni, Modular varieties with level 2 theta structure, Amer. J. Math. 116 (1994) 1489-1511.

[35] B. van Geemen, Siegel modular forms vanishing on the moduli space of curves, Invent. Math. 78 (1984) 329-349.

[36] B. van Geemen, G. van der Geer, Kummer varieties and moduli spaces of abelian varieties Am. J. Math. 108 (1986) 615-642.

[37] B. van Geemen, The Schottky problem and second order theta functions. Workshop on Abelian Varieties and Theta Functions (Spanish) (Morelia, 1996), 41-84, Aportaciones Mat. Investig., 13, Soc. Mat. Mexicana, Mxico, 1998. 